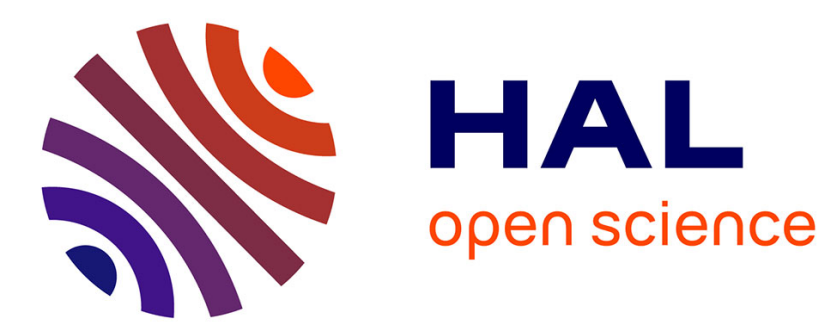

\title{
Nouvelles recherches sur l'aménagement du territoire angkorien à travers l'histoire
}

Christophe Pottier

\section{To cite this version:}

Christophe Pottier. Nouvelles recherches sur l'aménagement du territoire angkorien à travers l'histoire. Comptes-rendus des séances de l'Académie des inscriptions et belles-lettres, 2003, 147 (1), pp.427 449. halshs-02058821

\section{HAL Id: halshs-02058821 https://shs.hal.science/halshs-02058821}

Submitted on 6 Mar 2019

HAL is a multi-disciplinary open access archive for the deposit and dissemination of scientific research documents, whether they are published or not. The documents may come from teaching and research institutions in France or abroad, or from public or private research centers.
L'archive ouverte pluridisciplinaire HAL, est destinée au dépôt et à la diffusion de documents scientifiques de niveau recherche, publiés ou non, émanant des établissements d'enseignement et de recherche français ou étrangers, des laboratoires publics ou privés. 


\title{
Persée
}

\author{
http://www.persee.fr
}

\section{Nouvelles recherches sur l'aménagement du territoire angkorien à travers l'histoire}

\author{
Pottier, Christophe \\ Comptes-rendus des séances de l'année... - Académie des inscriptions et belles-lettres, Année 2003, Volume 147, \\ Numéro 1 \\ p. 427 - 449
}

Voir l'article en ligne

\begin{abstract}
Avertissement
L'éditeur du site «PERSEE » - le Ministère de la jeunesse, de l'éducation nationale et de la recherche, Direction de l'enseignement supérieur, Sous-direction des bibliothèques et de la documentation - détient la propriété intellectuelle et les droits d'exploitation. A ce titre il est titulaire des droits d'auteur et du droit sui generis du producteur de bases de données sur ce site conformément à la loi n`98-536 du $1 \mathrm{er} \mathrm{juillet} 1998$ relative aux bases de données.
\end{abstract}

Les oeuvres reproduites sur le site «PERSEE » sont protégées par les dispositions générales du Code de la propriété intellectuelle.

Droits et devoirs des utilisateurs

Pour un usage strictement privé, la simple reproduction du contenu de ce site est libre.

Pour un usage scientifique ou pédagogique, à des fins de recherches, d'enseignement ou de communication excluant toute exploitation commerciale, la reproduction et la communication au public du contenu de ce site sont autorisées, sous réserve que celles-ci servent d'illustration, ne soient pas substantielles et ne soient pas expressément limitées (plans ou photographies). La mention Le Ministère de la jeunesse, de l'éducation nationale et de la recherche, Direction de l'enseignement supérieur, Sous-direction des bibliothèques et de la documentation sur chaque reproduction tirée du site est obligatoire ainsi que le nom de la revue et- lorsqu'ils sont indiqués - le nom de l'auteur et la référence du document reproduit.

Toute autre reproduction ou communication au public, intégrale ou substantielle du contenu de ce site, par quelque procédé que ce soit, de l'éditeur original de l'oeuvre, de l'auteur et de ses ayants droit.

La reproduction et l'exploitation des photographies et des plans, y compris à des fins commerciales, doivent être autorisés par l'éditeur du site, Le Ministère de la jeunesse, de l'éducation nationale et de la recherche, Direction de l'enseignement supérieur, Sous-direction des bibliothèques et de la documentation (voir http://www.sup.adc.education.fr/bib/ ). La source et les crédits devront toujours être mentionnés. 


\title{
COMMUNICATION
}

\author{
NOUVELLES RECHERCHES \\ SUR L'AMÉNAGEMENT DU TERRITOIRE ANGKORIEN, \\ PAR M. CHRISTOPHE POTTIER
}

Angkor, Cité agraire par excellence en Asie du Sud-Est, se révèle tant par l'urbanisme de ses villes que par l'aménagement de son territoire. C'est dans cette optique et dans la continuité des travaux archéologiques entrepris à Angkor avant leur interruption prématurée en 1970, que je mène actuellement des recherches sur l'aménagement du territoire angkorien grâce au support permanent de l'École française d'Extrême-Orient et de ses directeurs, et avec l'aide généreuse de la Direction des Affaires culturelles au ministère des Affaires étrangères ${ }^{1}$.

\section{Pour une préservation et une compréhension globale de la région d'Angkor}

En 1992, le Cambodge et le site d'Angkor devenaient accessibles à la recherche après vingt ans de fermeture et l'ÉFEO y rouvrait son centre de Siem Reap. En décembre de la même année, le site et sa région étaient inscrits sur la liste du patrimoine mondial et l'UNESCO engageait une étude intitulée ZEMP pour Zoning Environmental Management Plan ou «Plan de zonage et de gestion de l'environnement d'Angkor dans le cadre de la région de Siem Reap ». Ce ZEMP devait fournir les bases pour analyser les ressources existantes et dégager des recommandations en vue d'une mise en œuvre d'un plan de protection du site d'Angkor et de développement de sa région. A l'occasion de cette étude, j'ai été amené à cartographier le patrimoine archéo-

1. Entre France et Cambodge, j'adresse mes sincères remerciements à MM. Bruno Dagens, Jacques Dumarçay et Claude Jacques qui m'ont généreusement fait bénéficier de leurs expériences d'Angkor, de leurs savoirs et de leurs interrogations. Mon apprentissage d'Angkor et les recherches que j'y ai entreprises leur sont pour beaucoup dus. 
logique de la région d'Angkor, soit une aire de $5000 \mathrm{~km}^{2}$. Ce travail a rapidement souligné l'existence de deux lacunes malgré le nombre des beaux et passionnants travaux déjà réalisés sur ce sujet. En premier lieu, la riche documentation archéologique rassemblée depuis plus d'un siècle n'avait été que rarement et partiellement cartographiée, leurs localisations géographiques étant souvent établies avec un faible niveau de précision ou sur des fonds cartographiques dorénavant inadaptés. J'ai alors eu recours à la télédétection, technique qui a largement fait ses preuves à Angkor depuis son introduction en 1930 par Victor Goloubew. J'ai repris l'ensemble des localisations des sites, me basant sur une nouvelle couverture photographique aérienne. Ce travail de télédétection préliminaire montra rapidement que le nombre de sites archéologiques repérés dépassait le double de celui des temples censés être connus par les inventaires antérieurs! Ceci confirmait ainsi l'ampleur d'un aspect déjà souligné en ces murs il y a plus de quarante ans par votre correspondant Bernard-Philippe Groslier, dont je voudrais ici saluer la mémoire, à savoir que de nombreux sites archéologiques demeuraient inédits aux abords même des capitales d'Angkor ${ }^{2}$. Mais la télédétection de 1992 permettait aussi l'identification des traces d'un tissu parcellaire ancien associé à de nombreux sites, offrant matière à des analyses morphologiques et à un développement prometteur de la compréhension des aménagements anciens à l'échelle de la région. Cette cartographie engagée comme un outil de protection et de gestion du patrimoine archéologique revêtait alors un évident intérêt scientifique.

Au moment d'être chargé de la direction de la Conservation d'Angkor, Bernard-Philippe Groslier avait souligné que le développement de la recherche archéologique à Angkor se devait d'associer deux « directions » distinctes mais complémentaires : une extension verticale (fouilles, sondages, étude des artéfacts et recherches sur l'environnement et son évolution) et une extension horizontale (prospection - essentiellement aérienne - pour replacer ces connaissances dans des problématiques territoriales) $)^{3}$. Bien entendu, ces deux « directions » venaient en com389.

2. B.-P. Groslier, « Nouvelles recherches archéologiques à Angkor », CRAI 1960, p. 337 -

3. B.-P. Groslier, "Our knowledge of Khmer civilization : a re-appraisal », Journal of the Siam Society 48 (1), Bangkok, 1960, p. 1-28. 
plément des piliers sur lesquels la connaissance d'Angkor a été patiemment bâtie depuis plus d'un siècle, croisant des sources pourtant limitées : les monuments, l'épigraphie et quelques rares sources extérieures. La tâche réalisée par de grands savants, tel votre regretté confrère George Cœdès, était considérable et se devait d'être poursuivie avec tous les moyens disponibles. Dans le contexte où les sources d'informations demeurent limitées, on ne s'étonnera pas que la tćlédétection ait été particulièrement mise en œuvre à Angkor et qu'elle ait été aussi fructueuse. Elle a en effet produit son lot d'informations supplémentaires et complémentaires depuis 1930 et les travaux pionniers de Victor Goloubew ${ }^{4}$. En repérant les traces d'aménagements inédits associés à des temples bien connus, l'un des premiers apports de la télédétection a consisté en l'intégration de l'environnement immédiat pour la compréhension de ces temples dans un contexte urbain. C'est ainsi que de larges enceintes étaient repérées, mais aussi des canaux, des chaussées, des digues, autant de vestiges qui révélaient un réseau complexe à Angkor et autour de ses capitales successives 5 . La télédétection et les campagnes de prospection au sol qu'elle motivait jetaient alors les prémisses de l'étude du territoire angkorien dont témoignent encore par exemple les levés toujours fiables et détaillés du talentueux architecte Georges Trouvé . Ccs informations engendrèrent aussitôt diverses hypothèses, à une époque particulièrement riche en hypothèses suite aux chamboulements qu'avait connus la chronologie angkorienne grâce aux études de Philippe Stern et de George Cœdès ${ }^{7}$. Dans ce contexte où des pans entiers de l'histoire d'Angkor étaient déstabilisés, ces nouvelles informations se

4. A propos de l'histoire de la télédétection à Angkor : C. Pottier « Mapping Angkor : for a new appraisal of the Angkor region ", Space applications for heritage conservation, European Space University, Noordwijk, Publ. électronique, 2002.

5. V. Goloubew, "Reconnaissances aériennes au Cambodge ", Bulletin de l'EFEO 36 (2), Hanoi, 1936, p. 465-477. V. Goloubew, « Recherches aériennes », Bulletin de l'EFEO 37 (2), Hanoi, 1937, p. 655-656. H. Parmentier, « Examen du levé d'avion du parc d'Angkor par le Capitaine Gouet les 24-26 janvier 1933 à 9 heures à l'altitude de $1300 \mathrm{~m}$ », Bulletin de l'EFEO 33 (2), Hanoi, 1933, p. 1111-1116.

6. G. Trouvé, "Chronique. Chaussées et canaux autour d'Angkor Thom », Bulletin de l'ÉFEO 33 (2), Hanoi, 1933, p. 1120-1128. Voir aussi sa carte d'ensemble du groupe d'Angkor publiée sous la planche $\mathrm{n}^{\circ} 1$ dans Documents topographiques de la Conservation d'Angkor, J. Dumarçay (Introduction), C. Pottier (Préparés par), MÉFEO 21, Paris, 1993.

7. P. Stern, Le Bayon d'Angkor Thom et l'évolution de l'art Khmer, Annales du musée Guimet, tome 47, Paris, 1927. G. Codès, « La date du Bayon », Bulletin de l'EFEO 28 (1-2), Hanoi, 1928, p. 81-112. 
révélaient particulièrement appropriées pour s'interroger sur l'identification et la constitution de certaines capitales : elles aboutirent alors sur le terrain au lancement de plusieurs campagnes de fouilles, en particulier aux abords d'Angkor Thom et du Phnom Bakheng à la recherche des Yaçodharapura ${ }^{8}$.

Interrompue lors de la seconde guerre mondiale, la télédétection connaît un nouvel élan avec les travaux de Bernard-Philippe Groslier dès le début des années 50 , au début dans le delta du Mékong, puis rapidement remontant vers le nord, à Angkor même où le même chercheur s'attache en premier lieu aux environs proches de la dernière capitale, Angkor Thom. Il y précise le détail du complexe écheveau de canaux qui alimentaient et drainaient cette ville et intègrent ses observations à une analyse serrée des descriptions portugaises du XVI $\mathrm{X}^{\mathrm{e}}$ siècle ${ }^{9}$. Mais dès la fin des années 1950, ces travaux de télédétection l'entraînent à aborder une échelle plus vaste et à interroger les rapports étroits que les capitales entretenaient avec leur environnement et leur région, le terroir angkorien. Bernard-Philippe Groslier approfondit patiemment et peaufine ce thème d'étude dans plusieurs articles et publie finalement en 1979 dans le Bulletin de l'ÉFEO une synthèse qui est restée justement célèbre sous le nom de « La Cité Hydraulique ${ }^{10}$. Malheureusement, les recherches de terrain étaient déjà interrompues à Angkor, depuis que la région avait été brutalement plongée dans le chaos dès 1970 .

Dans le contexte de la réouverture d'Angkor il y a dix ans, la complémentarité des approches horizontales et verticales proposées par Bernard-Philippe Groslier dès 1959 ne me semblait pas avoir été fondamentalement remise en cause par les nombreuses avancées réalisées entre temps. Au contraire, elles avaient souvent confirmé la justesse de ses vues. La nécessité d'une double approche pouvait donc encore se poser dans des termes assez proches, en comptant aussi évidemment avec le renouvellement des techniques. Mais une direction verticale supposait des

8. Victor Goloubew mène de 1931 à 1937 des campagnes de prospections et de fouilles dont les résultats sont publiés dans les Bulletins de l'EFEO. Cf. C. Pottier, «A la recherche de Goloupura ", Mélanges du centenaire de l'EFEO, Bulletin de l'EFEO 87, vol. 1, 2001, Paris, p. 79-107.

9. B.-P. Groslier, Angkor et le Cambodge au XVre siècle d'après les sources portugaises et espagnoles, Annales du musée Guimet, Bibliothèque d'étude 63, Paris, 1958.

10. B.-P. Groslier, «La cité hydraulique angkorienne : exploitation ou surexploitation du sol ? », Bulletin de l'ÉFEO 66, 1979, Paris, p. 161-202. 
moyens d'action et une accessibilité qui n'étaient pas alors réunis en 1992. J'ai donc opté en un premier temps pour une approche horizontale en entreprenant de développer le premier travail préliminaire pour l'UNESCO, en l'approfondissant sur une aire plus réduite et plus adaptée pour aborder précisément l'étude diachronique de l'aménagement du territoire angkorien.

\section{Première phase : cartographie et analyse de la zone sud d'Angkor}

\section{CARTOGRAPHIE}

De 1993 à 1999, la première phase de cette étude s'est limitée à la « zone sud » d'Angkor, soit $600 \mathrm{~km}^{2}$ compris entre Angkor Thom, ses deux principaux baray et le lac Tonlé Sap, cadre spatial et chronologique correspondant au berceau des premières phases de l'histoire d'Angkor ${ }^{11}$. L'établissement d'une cartographie détaillée et d'un nouveau corpus de sites archéologiques de cette zone était toutefois la condition préalable pour engager une étude de l'aménagement ancien de la région. Cette cartographie s'est basée sur un affinement de mes précédents travaux de télédétection, la recherche de références bibliographiques éparses et des vérifications systématiques de terrain, seules à même de confirmer la validité des repérages (fig. 1). L'établissement de ce corpus et la réalisation de la cartographie a bénéficié de la conjonction de divers facteurs favorables, à commencer par l'existence d'un remarquable fonds topographique au $10000^{\mathrm{e}}$ que Bernard-Philippe Groslier avait justement fait réaliser au début des années 1960 dans le but de constituer un atlas archéologique $^{12}$. D'autre part, les nouvelles prises de vues aériennes de 1992 témoignaient de vastes déforestations récentes et recelaient un potentiel que ma première étude n'avait fait qu'effleurer. Par ailleurs, un programme d'informatisation et d'indexation du fonds documentaire des archives de l'ÉFEO à Paris avait été entrepris en 1990 sous la direction de Bruno Dagens et rendait

11. Cette première phase de la recherche a fait l'objet en 1999 d'un Doctorat à l'Université de Paris III sous la direction de Bruno Dagens, intitulé : Carte archéologique de la région d'Angkor - Zone sud.

12. Les 25 minutes topographiques ont été publiées (planches 2 à 26) dans Dumarçay et Pottier, op. cit. n. 6. 
alors possible l'exploitation de ces ressources cruciales, même depuis Siem Reap, sur le terrain. Enfin il faut souligner que, étant engagé en tant qu'architecte sur des restaurations planifiées sur plusieurs années et donc résidant en permanence sur le site grâce à l'appui constant de l'ÉFEO et du ministère des Affaires étrangères, je pouvais envisager la réalisation de prospections systématiques du terrain étudié, en m'adaptant plus aisément à des conditions de sécurité mouvantes à cette époque.

Au final, le corpus qui a ainsi été rassemblé comprenait près de deux tiers de sites inédits : au total, 527 sites ponctuels ont été répertoriés, dont 327 inédits. La figure 2 présente la localisation de ces sites, dissociant en jaune les sites inédits des sites en rouge repérés et inventoriés par mes prédécesseurs (Aymonnier, Lunet de Lajonquière, Marchal, Trouvé et Groslier).

\section{PREMIERS RÉSULTATS ET HYPOTHÈSES}

Cette cartographie et ce corpus ont alors offert les assises nécessaires pour approfondir l'étude des divers types d'ouvrages hydrauliques, dont les fameux baray, et pour élaborer des hypothèses sur la répartition de l'habitat et des zones de culture. De même l'étude simultanée des vestiges de constructions anciennes et des tissus parcellaires m'a amené à engager une réflexion sur la chronologie et la constitution des capitales, et sur leur insertion dans des contextes préexistants ${ }^{13}$. A travers plusieurs cas, la remise en perspective historique de cette cartographie précise l'organisation des agglomérations et leurs extensions dans le territoire. Ainsi peut-on consolider les vues de BPG relatives à la localisation de sites protohistoriques en rive du lac Tonlé Sap et des terres inondables, sur la «rive archéologique ». A Hê Phka comme au sud de Rolûos par exemple, l'analyse spatiale montre que ces sites qui présentent des configurations complexes de terre-pleins artificiels singulièrement organisés se retrouvant par ailleurs dans les ensembles préangkoriens de la région, peuvent constituer autant de cas de superposition, voire de continuité, entre les installations protohistoriques et les premières périodes

13. C. Pottier, "About Yaçovarman's Buddhist açrama in Angkor», The Buddhist Monastery: A cross-cultural survey, P. Pichard et F. Lagirarde (éd.), ÉFEO, Paris, p. 199-208. C. Pottier, "Élaboration d'une carte archéologique de la région d'Angkor ", Southeast Asian Archaeology 1994, vol. I, P.-Y. Manguin (éd.), 1998, Leiden, p. 179-194. 


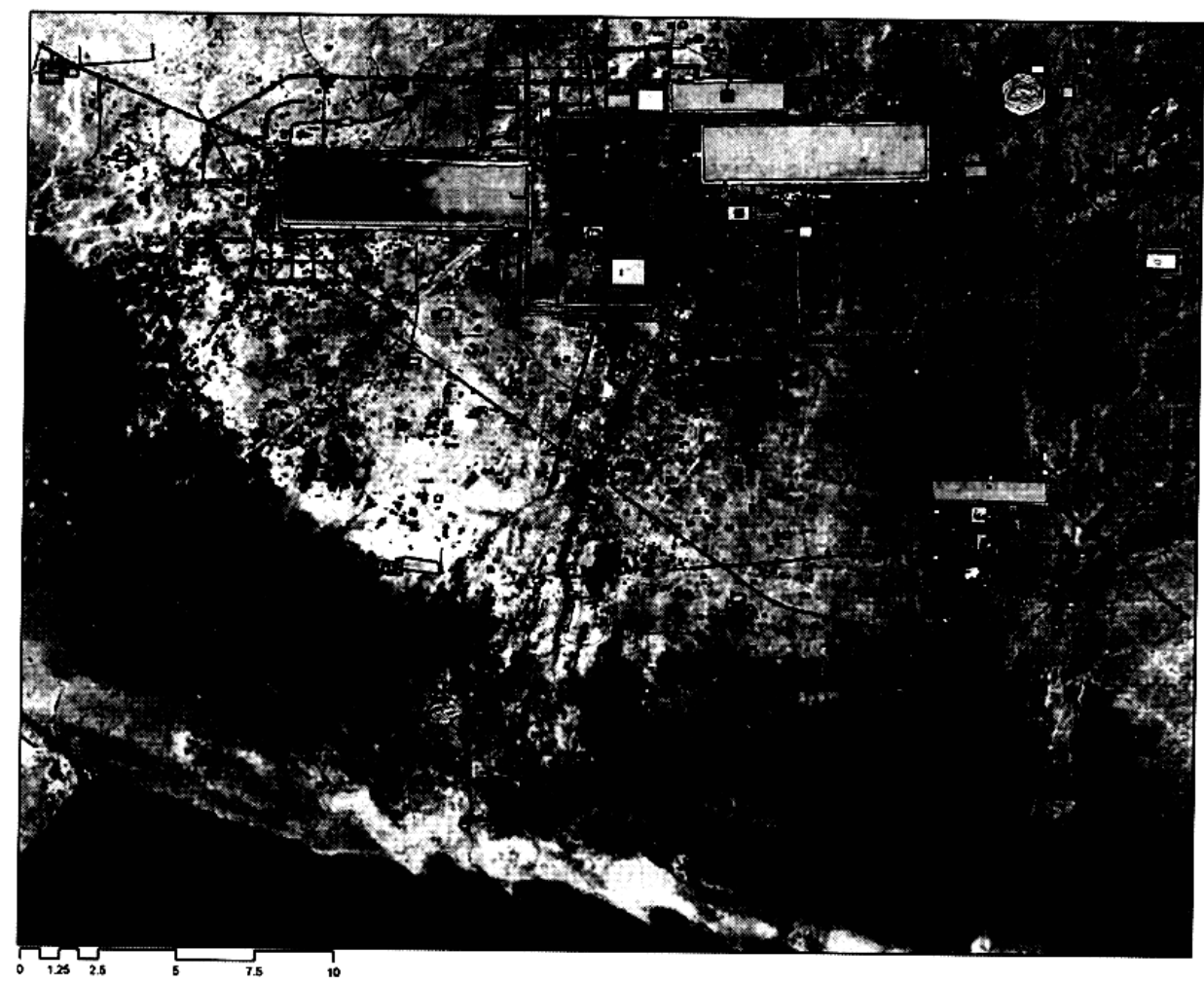

Fig. 1. - Sites et structures archéologiques repérés dans la zone sud d'Angkor (EFEO/C. Potier 1999).

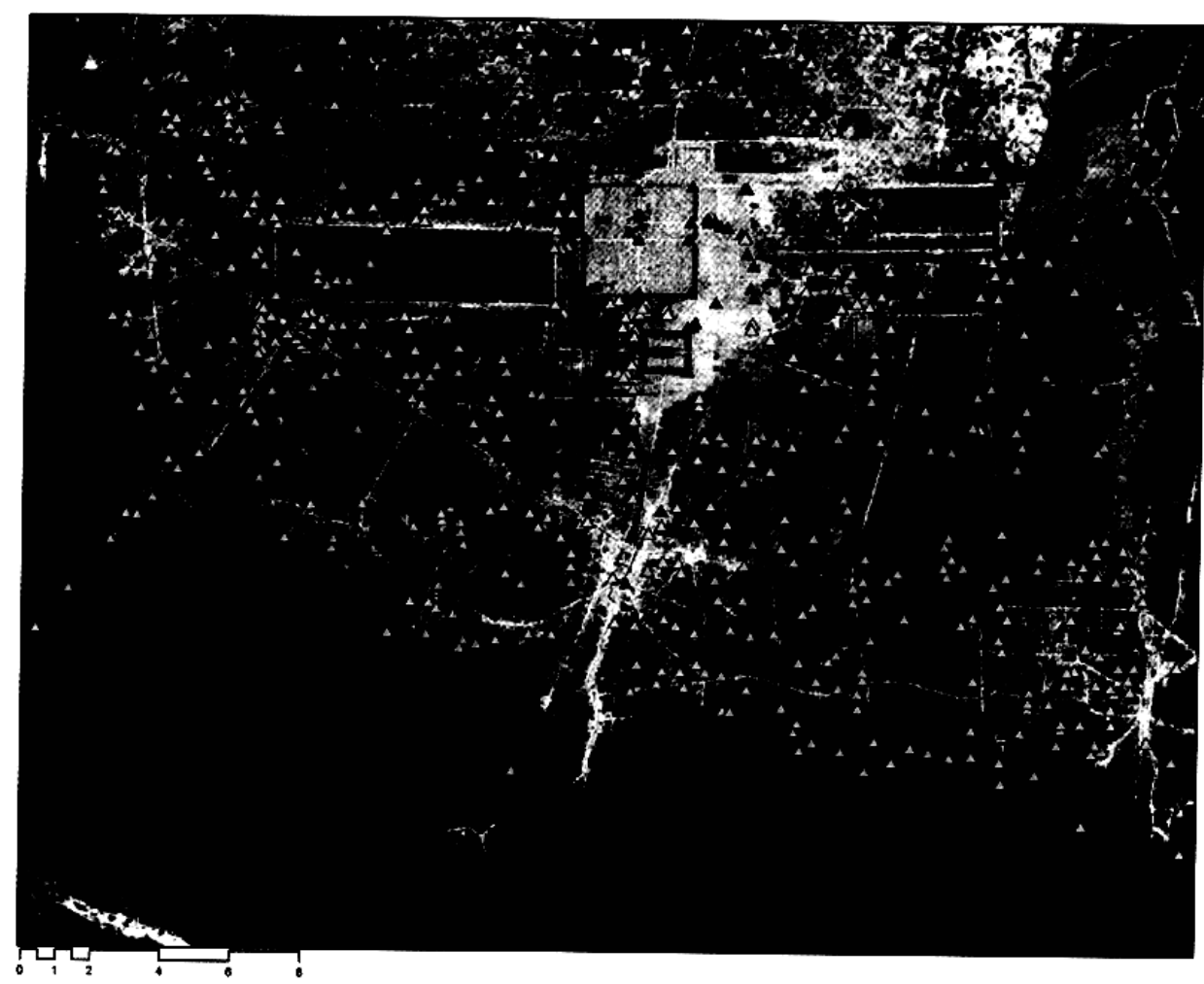

Fig. 2. - Sites archéologiques repérés dans la zone sud d'Angkor. Les sites inventoriés sont en rouge, les inédits en jaune (EFEO/C. Potier 1999). 


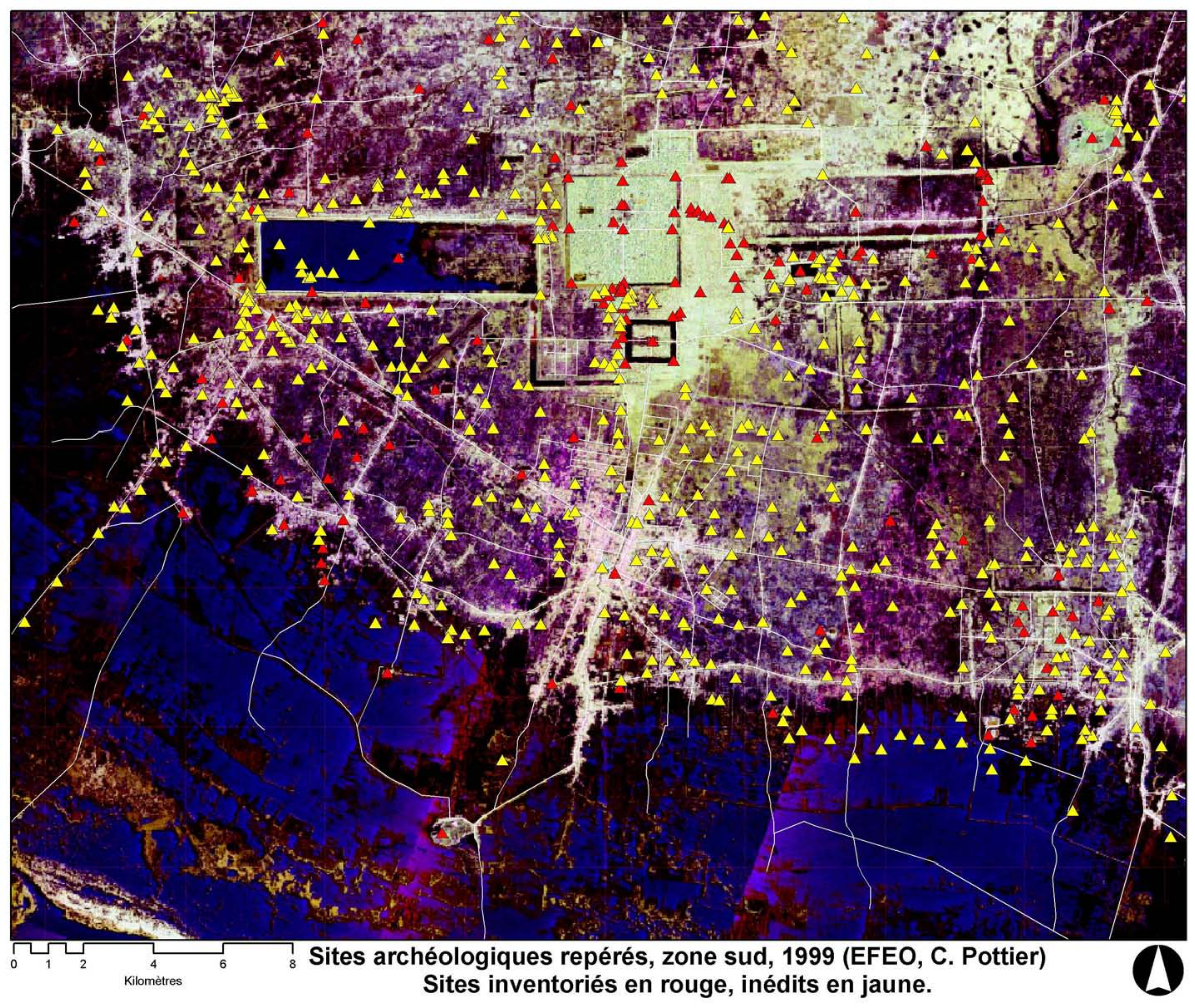


dites historiques, avec l'installation de sanctuaires de culte brahmanique (fig. 3). Mais divers éléments nouvellement cartographiés permettent aussi de reconsidérer l'histoire de l'aménagement de cette région d'Angkor et ses caractéristiques principales en précisant, voire en modifiant parfois sensiblement, la remarquable et à ce jour inégalée synthèse proposée par Bernard-Philippe Groslier dans « La cité hydraulique »14. En particulier, deux moments de ruptures distincts ont semblé pouvoir être dégagés entre les premières occupations humaines et le $\mathrm{x}^{\mathrm{e}}$ siècle : le premier marque l'apparition de la cité angkorienne correspondant à une centralisation et à une géométrisation des occupations humaines, le second celle du territoire hydraulique avec la mise en place d'un système hydraulique et d'un aménagement planifié des abords en aval de cités largement ouvertes. On croit en particulier pouvoir observer la première rupture, la création de la cité angkorienne, tant dans la région d'Ak Yum qu'à Rolûos où s'opère un glissement vers le nord depuis les premières installations proches de Trapéang Phong.

Mais ce glissement s'accompagne aussi de deux faits essentiels, celui d'une géométrisation et d'une concentration des aménagements autour d'un point focal marqué par le temple de forme pyramidale, caractéristiques que l'on peut suivre tant à Ak Yum qu'à Bakong... et qui perdureront lors des siècles suivants à tel point qu'elles peuvent être considérées comme les deux premiers principes de l'urbanisme à Angkor. Et même dans le cas de sites bien connus comme le Bakong (fig. 4), la cartographie et la télédétection permettent encore d'y relever les éléments attestant des aménagements qui accompagnaient le temple, par exemple en montrant l'existence de réseaux de bassins tant à l'intérieur de la douve périphérique qu'à l'extérieur le long des deux chaussées axiales principales, attestant d'une conception monumentale rigoureuse dépassant l'enceinte du temple et étroitement liée au territoire. Elles y montrent d'ailleurs aussi que ce schéma de bassins a été altéré à au moins une occasion lors de l'installation de l'emprise de Prah Kô. Ceci ne serait que détail si cette antériorité du Bakong et de ses bassins sur Prah Kô n'était pas en apparence contreditc par plusicurs inscriptions d'Indravarman $\mathrm{I}^{\text {er }}$ sur la foi desquelles on a attribué à ce roi toute la paternité - et l'ini-

14. B.-P. Groslier, op. cit. n. 10. 


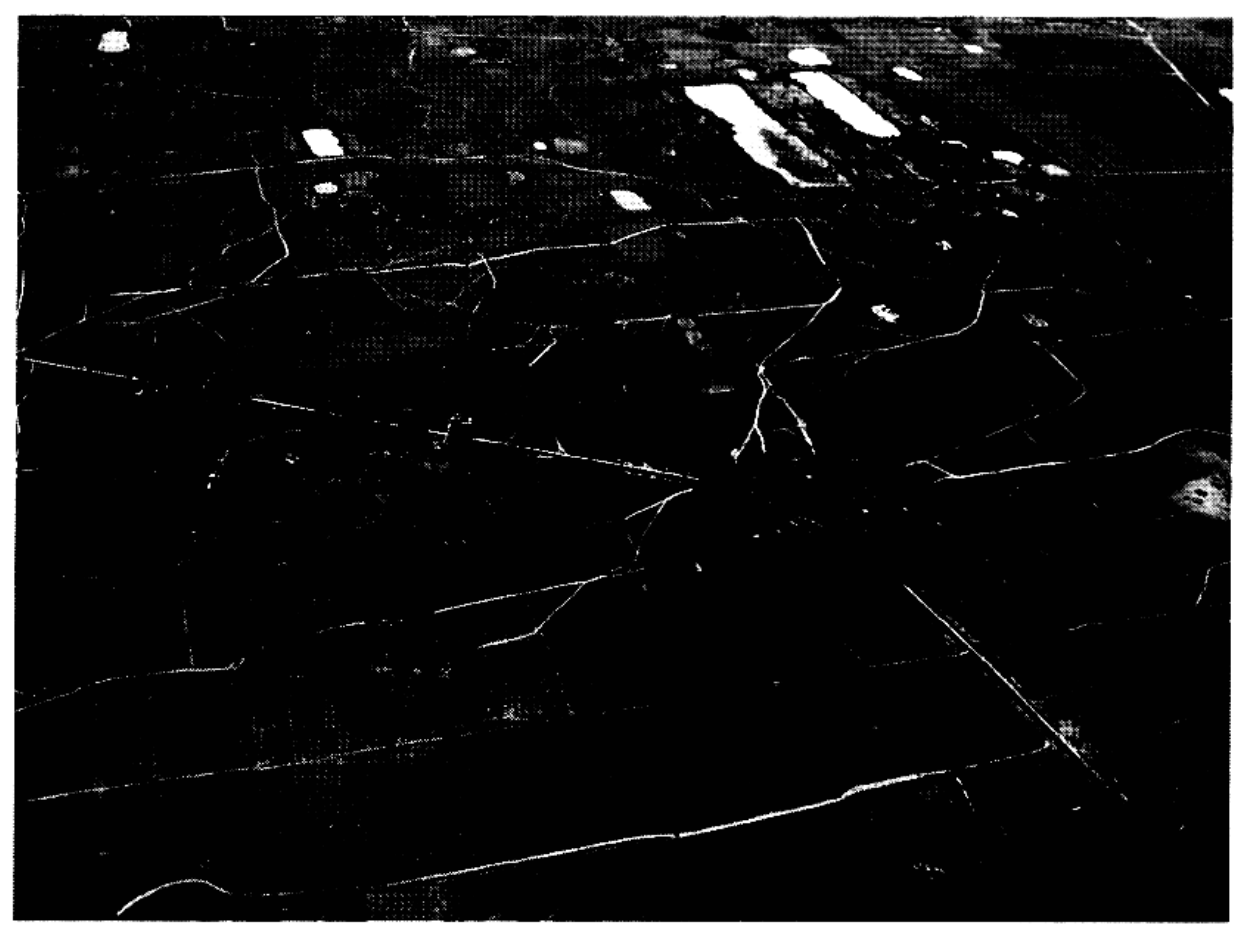

FIG. 3. - Terre-pleins et bassins dans la région Sud de Rolûos près de Trapéang Phong. Vue vers le sud-est.

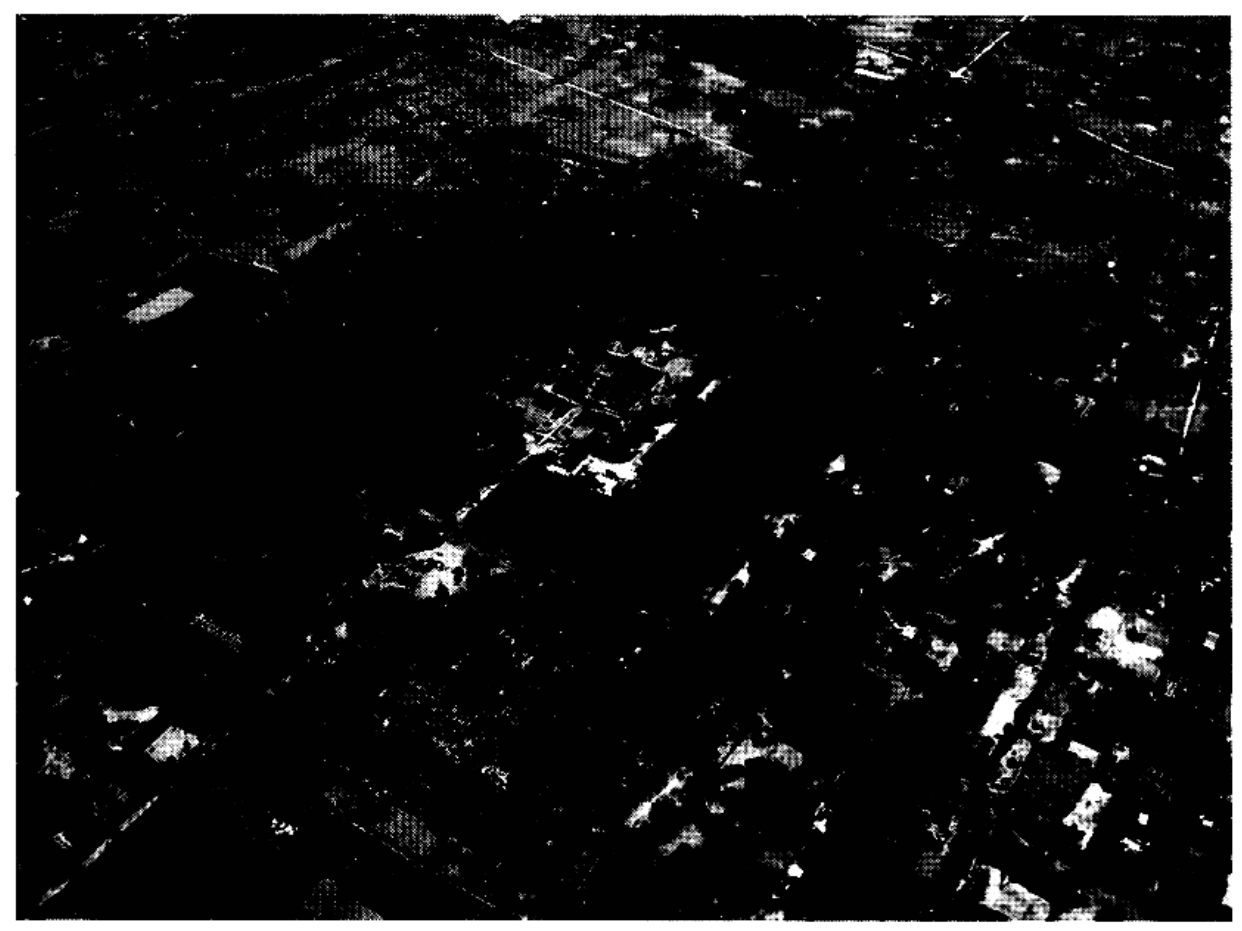

Fig. 4. - Le temple et les douves du Bakong à Rolûos. Vue vers le sud-ouest. 


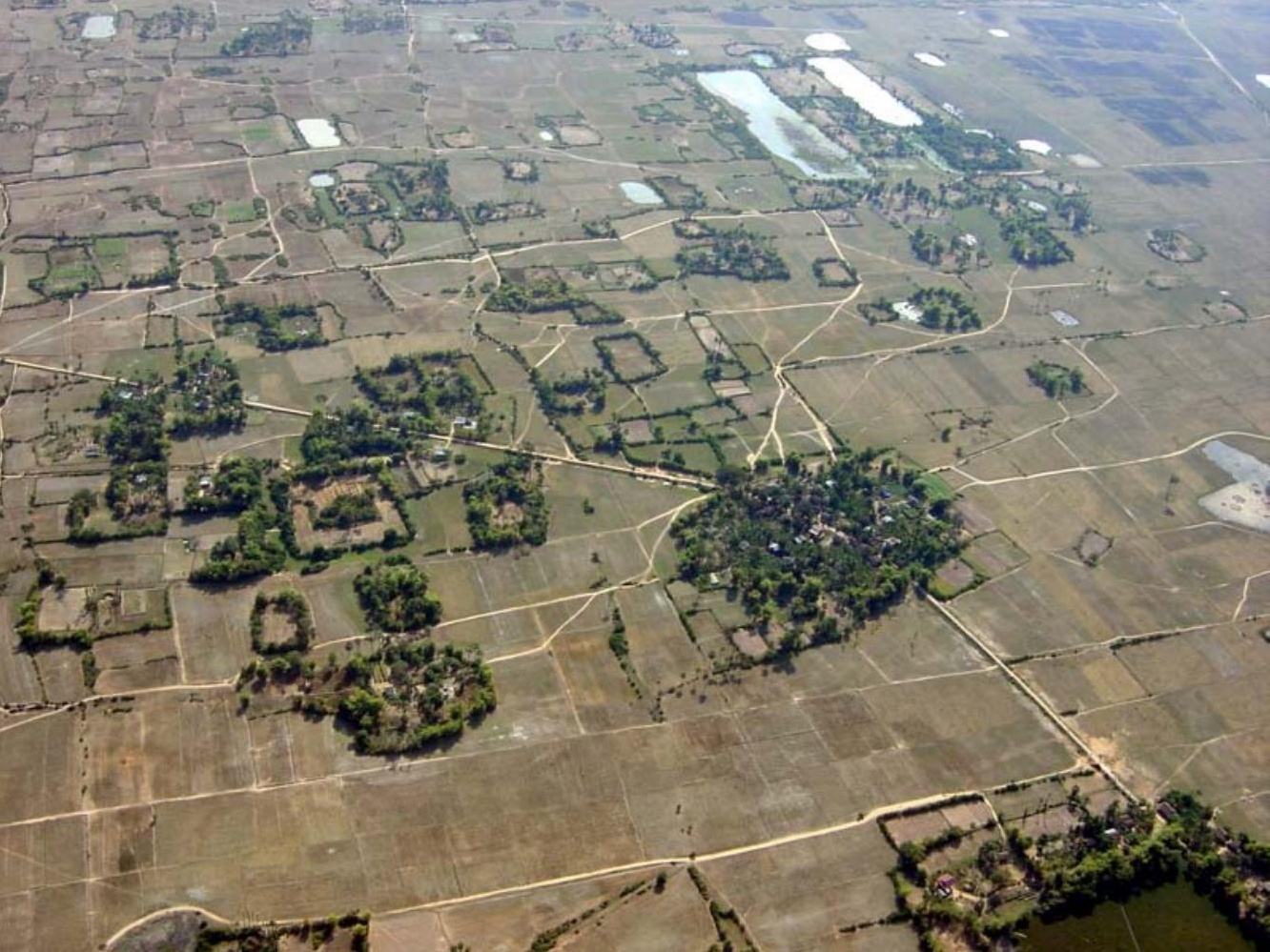


for.

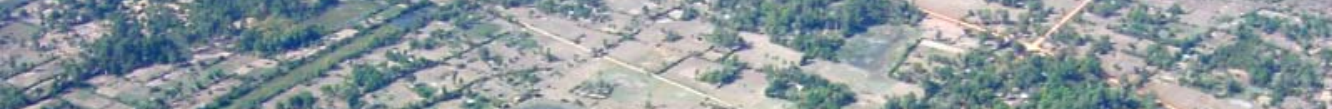

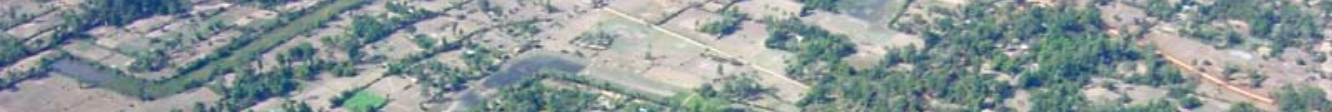

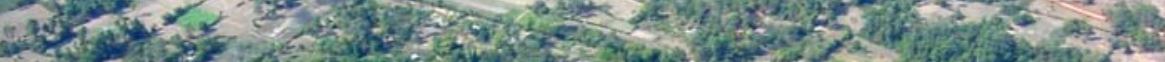

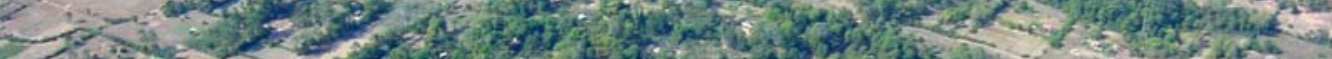

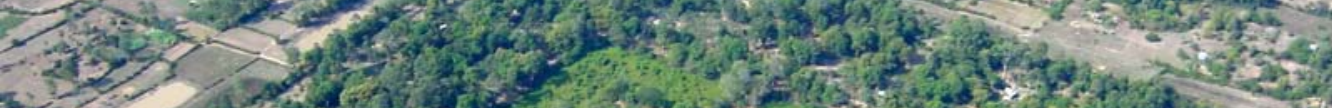

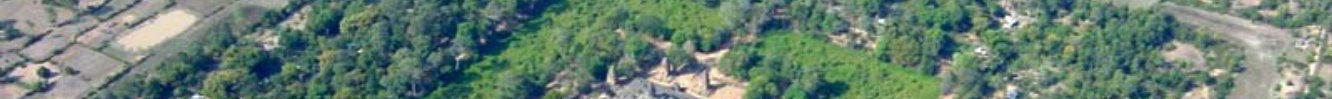

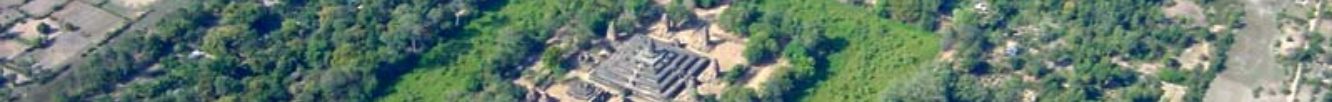
20. 2. -

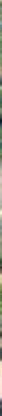


tiative - de ces deux temples et donc de la première capitale angkorienne $^{15}$. Il apparaît donc ainsi que l'on doit envisager que le Bakong ait pu être conçu et les travaux largement engagés par un des prédécesseurs d'Indravarman I $^{\text {er }}$ et que l'on doit ainsi du même coup situer l'apparition de la cité angkorienne à Rolûos dans la première moitié du $\mathrm{IX}^{\mathrm{e}}$ siècle, voire avant ${ }^{16}$.

Une des autres hypothèses issues de l'analyse de cette nouvelle cartographie porte sur la forme des établissements urbains dc la région d'Angkor. Fortement influencées - consciemment ou non - par la dernière capitale Angkor Thom, ville murée dans un carré parfait de $3 \mathrm{~km}$ de côté, nombreuses sont les recherches qui ont tenté de retrouver ce schéma idéal ailleurs dans les environs. La cartographie tend à promouvoir une image opposée où l'urbanisme angkorien peut être caractérisé par son aspect ouvert jusqu'au $\mathrm{XI}^{\mathrm{e}}$ siècle. Je citerai tout d'abord le cas de Bantay Chhœu, une première cité carrée envisagée par plusieurs auteurs dont Bernard-Philippe Groslier, englobant plusieurs sites préangkoriens aux abords du baray occidental ${ }^{17}$. Télédétection et prospections s'accordent pour ne voir dans l'hypothétique enceinte de cette Bantay Chhœu qu'une partie d'un vaste réseau de canaux associés au baray occidental et donc très vraisemblablement contemporain de celui-ci au XI ${ }^{\mathrm{e}}$ siècle $^{18}$. Un second exemple porte sur l'hypothèse émise par Victor Goloubew de l'existence autour du Phnom Bakheng, fondé par Yaçovarman ${ }^{\text {er }}$, d'une ville ceinte d'une vaste double digue de $4 \mathrm{~km}$ de côté... mais dont ne subsisterait ou n'aurait jamais existé que l'angle sud-ouest. J'ai eu récemment l'occasion de me pencher sur cette hypothèse très largement acceptée depuis 70 ans, hypothèse qu'Henri Marchal, collègue et collaborateur de Victor Goloubew, avait amicalement - mais néanmoins sceptiquement - nommée Goloupura ${ }^{19}$. Ici, la

15. P. Stern, « Diversité et rythme des fondations royales khmères », Bulletin de l'ÉFEO 47 (2), Paris, 1954, p. 649-687.

16. C. Pottier, « Notes sur le Bakong et son implantation », Bulletin de l'EFEO 83, chroniques, 1996, Paris, p. 318-326. De nouveaux éléments recueillis tant lors de notre dernière campagne de fouilles de 2004 que dans le cadre de notre collaboration avec l'Université de Sydney confirment encore cette première analyse : D. Penny, C. Pottier, R. Fletcher, M. Barbetti, D. Fink et $Q$. Hua, «A palynological record of vegetation and land-use change from Angkor - Hariharalaya ", Antiquity, York (à paraître).

17. B.-P. Groslier, op. cit. n. 10, p. 167, cartes 2 et 3.

18. C. Pottier, "Some evidence of an inter-relationship between hydraulic features and rice field patterns at Angkor during ancient times ", The Journal of Sophia Asian Studies, $\mathrm{n}^{\circ} 18,2000$, Tokyo, p. 99-119.

19. C. Pottier, op. cit. n. 19. 


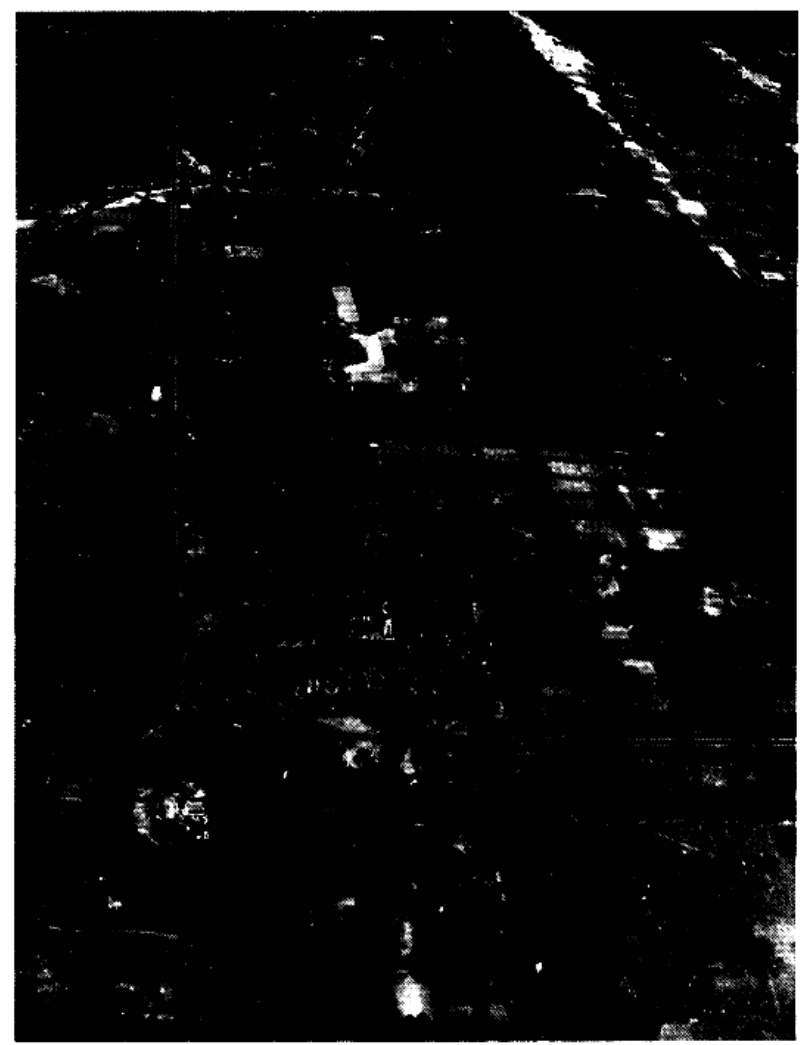

Fig. 5. - Pre Rup et ses environs. Vue vers l'ouest.

télédétection et les prospections associées à quelques carottages ont permis de confirmer l'opinion de Bernard-Philippe Groslier qui doutait de l'existence de cette Goloupura, et de proposer une datation nettement plus tardive aux vestiges précédemment compris comme enceinte de ville. J'évoquerai enfin un troisième et dernier cas qui est à mon avis exemplaire de cet urbanisme ouvert; il s'agit de la ville établie aux environs du temple de Pre Rup au sud du baray oriental, lors du règne de Ræjendravarman $I^{\text {er }}$ (fig. 5). On y observe de nombreux vestiges, tertres, bassins et chaussées, remarquablement intégrés dans une organisation cohérente suggérant même la localisation du Palais royal de l'époque édifiée par l'architecte Kavindrærimathana ${ }^{20}$. Mais d'enceinte, nulle trace ! L'analyse du système parcellaire ancien

20. G. Cœdès, «Les inscriptions de Bat Cum (Cambodge) », Journal Asiatique 10 (12), Paris, 1908 p. 213-254. C. Jacques, traduction non publiée des inscriptions de Bat Chum, document dactylographié. 


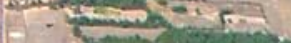

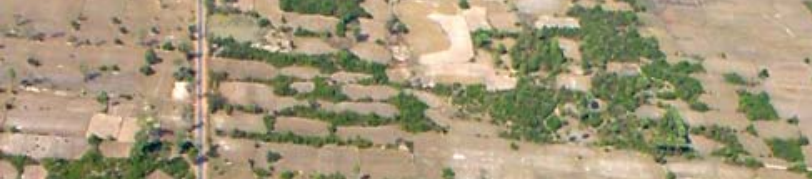

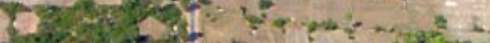

3e.

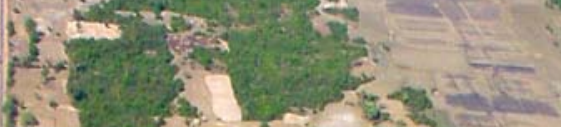

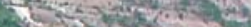

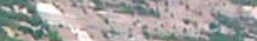

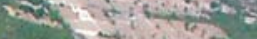
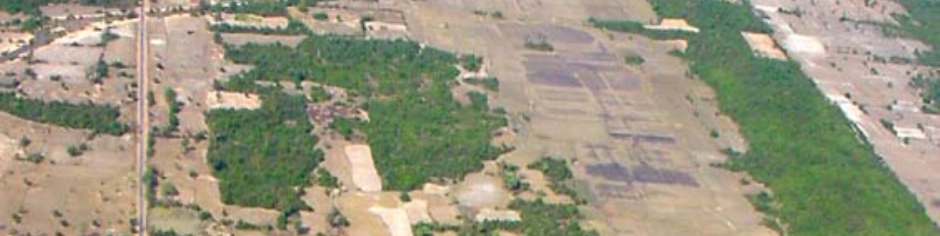

\section{sexisents

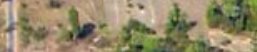

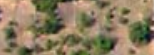

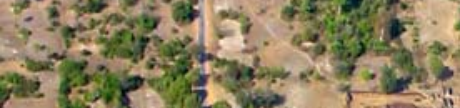

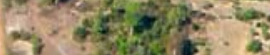

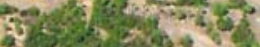

3.

\section{2.}

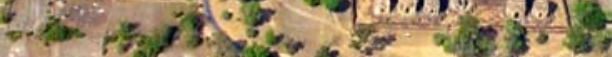

Q

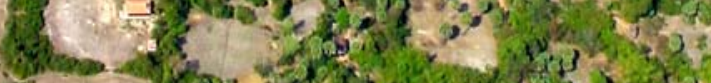

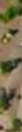

Hex

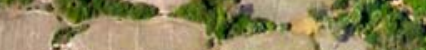

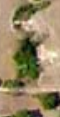

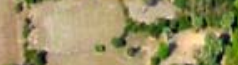

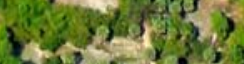

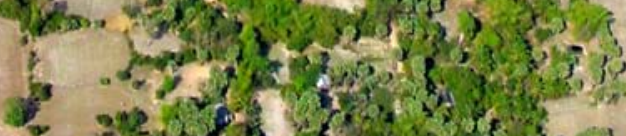

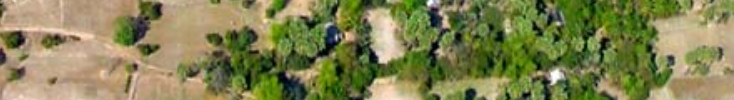


confirme encore ce constat, montrant que l'urbain ne se distingue que par une densification s'estompant progressivement vers le territoire dans un remarquable continuum (fig. 6).

\section{SECONDE PHASE : EXTENSION DE LA CARTOGRAPHIE ET MISSION} ARCHÉOLOGIQUE

Au terme de cette première phase et sur la base des enseignements apportés par l'examen de cette zone test, j'ai entrepris de poursuivre et de développer cette recherche tant d'un point de vue spatial que méthodologique. Car si l'approche horizontale a bien montré un vaste potentiel pour aborder les problématiques liées aux aménagements anciens de la région et pour développer des hypothèses sur leur évolution, ces interprétations spatiales et ces chronologies relatives nécessitent d'être vérifiées et consolidées par des informations stables issues de l'approche verticale. La seconde phase de cette recherche a donc été développée pour intégrer cette complémentarité, associant à l'extension de la cartographie et à la poursuite des analyses spatiales un volet spécifiquement dévolu à des recherches en profondeur qui font l'objet d'une mission archéologique financée par la Commission archéologique du ministère des Affaires étrangères. Les travaux ont donc été engagés parallèlement sur deux niveaux en 1999.

\section{Télédétection, prospections et analyses spatiales}

La poursuite de l'étude territoriale sur l'ensemble de la périphérie d'Angkor constitue l'objet premier de ma recherche qui s'inscrit dans le cadre du programme quadriennal de l'équipe pluridisciplinaire de l'ÉFEO « Angkor - De l'espace du temple à l'aménagement du territoire ». Une coopération a été engagée depuis trois ans avec Roland Fletcher, professeur au département d'archéologie de l'Université de Sydney, pour développer un volet de cette recherche spécifiquement centré sur l'expansion de l'urbanisation de la région d'Angkor ${ }^{21}$.

21. Cette collaboration se déroule dans le cadre du «Greater Angkor Project », coopération entre l'Université de Sydney, APSARA (Autorité pour la Sauvegarde et l'Aménagement de la Région d'Angkor) et l'ÉFEO. Voir : Greater Angkor Project, «Redefining Angkor : structure and Ecology in the largest, low density urban complex of the pre-industrial world », UDAYA n ${ }^{\circ}$ 4, APSARA, Phnom Penh, 2003, p. 107-125. 


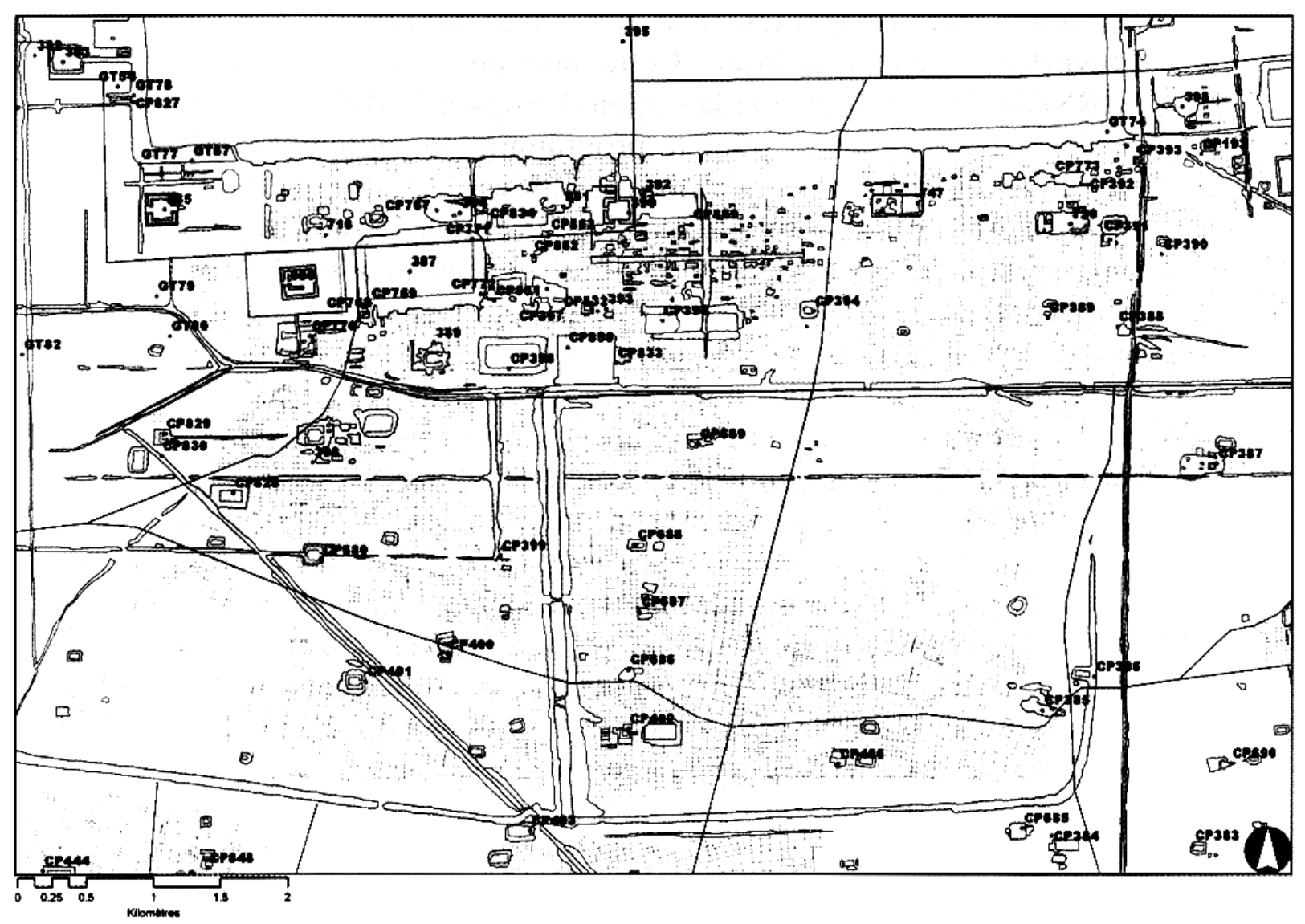

Fig. 6. - Sites archéologiques et tracés parcellaires dans la région de Pre Rup au sud du baray oriental (EFEO/C. Potier 1999).

Notre recherche s'applique désormais à étendre vers le nord la couverture de la cartographie archéologique et à y cerner les phases de développements plus étendus et, en particulier, à analyser les périodes postérieures au $\mathrm{X}^{\mathrm{e}}$ siècle peu ou très partiellement sensibles dans la zone méridionale étudiée auparavant. Cette zone septentrionale apparaît comme particulièrement importante pour une meilleure compréhension des zones proches des baray angkoriens, éléments majeurs de certaines stratégies territoriales angkoriennes (fig. 7). La méthodologie associe désormais des outils informatiques plus puissants et l'ensemble des données sont intégrées dans un système d'informations géographiques (SIG). Profitant de la méthodologie mise au point sur la zone sud, les travaux se basent toujours solidement sur la télédétection, utilisant encore les couvertures photographiques 


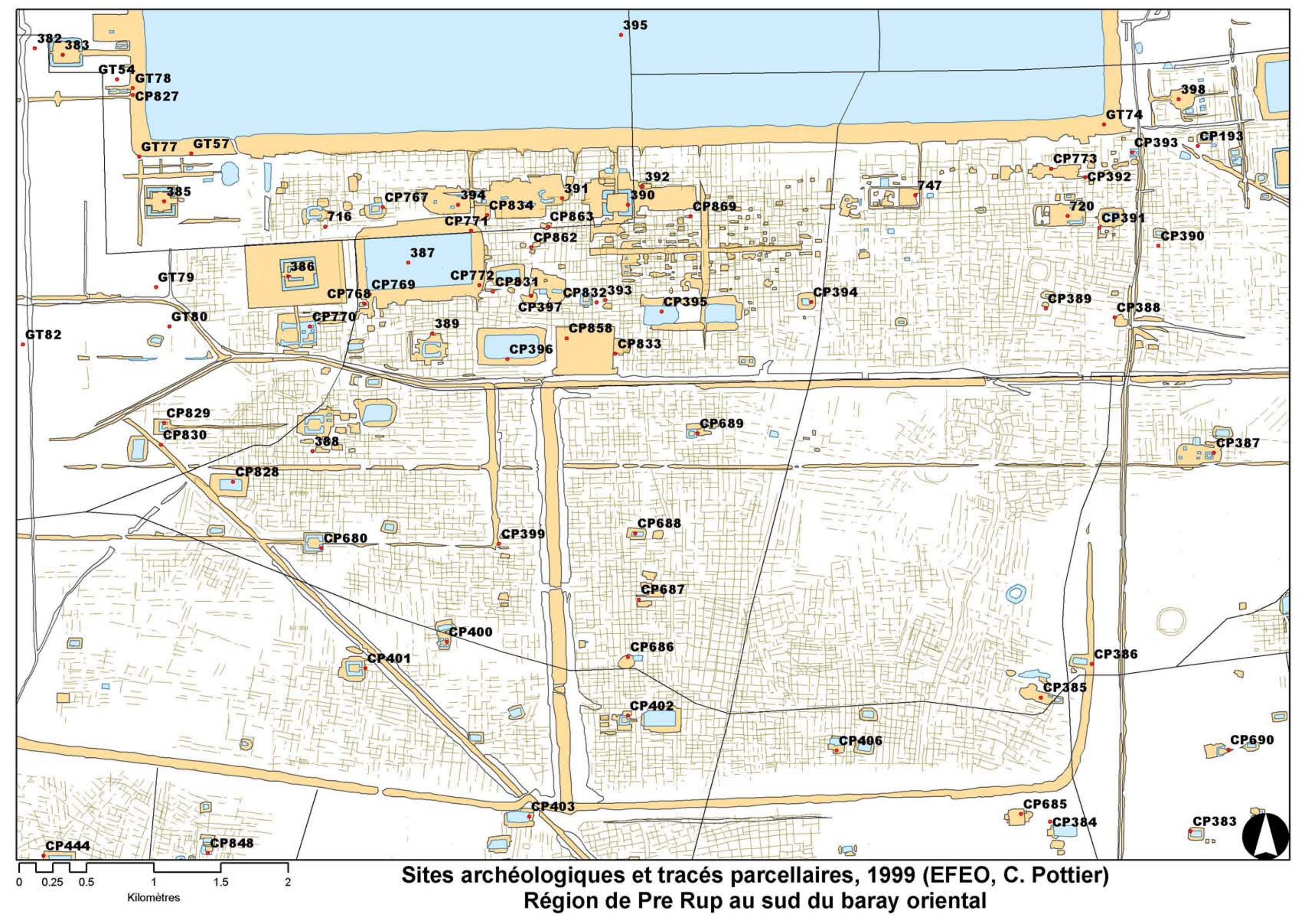


aériennes stéréoscopiques, mais désormais renforcées par une couverture radar spécifique. Cette nouvelle couverture radar AIRSAR de l'ensemble de la région d'Angkor $\left(7000 \mathrm{~km}^{2}\right)$ a été réalisée spécifiquement pour le programme en septembre 2000 par la NASA/JPL ${ }^{22}$. Les « clichés », rendus disponibles à la fin d'année 2001, ont été intégrés au SIG et enrichissent désormais nos travaux de télédétection en offrant le premier modèle tridimensionnel de l'ensemble de cette région. Remarquons que la télédétection revêt une importance encore plus forte qu'au Sud puisqu'il sera sans doute impossible de vérifier au sol toute la zone extrême nord (au pied du Phnom Kulen) à cause de la présence de zones encore minées ou couvertes d'une brousse impénétrable. Toutefois, l'expérience acquise en télédétection permettra de limiter les marges d'erreur. Et le projet bénéficie par ailleurs de l'aide généreuse d'un particulier ${ }^{23}$ qui met gracieusement à disposition un ULM et ses remarquables qualités de pilote pour la réalisation de prospections aériennes particulièrement intéressantes dans des zones inaccessibles au sol ou le long de structures linéaires difficiles à suivre sur le terrain.

Profitant de la méthodologie mise au point sur la zone sud, les travaux associent toujours recherche documentaire, télédétection préalable, prospections guidées au GPS, vérifications de terrain, collectes d'échantillons d'artefacts en surface et carottages. Outre quelques éléments spectaculaires, telle une superbe stèle inscrite inédite découverte au Prasat Phnom Dei ${ }^{24}$, ce travail apporte des éléments parfaitement cohérents avec ceux dégagés dans la zone symétrique au sud. On note pourtant plusieurs différences, dans la densité des structures linéaires par exemple, mais aussi dans la proportion de sites ponctuels inédits qui y est nettement plus importante. En effet, cette proportion était environ de deux tiers dans la zone sud; on l'estime désormais à environ $85 \%$ dans la zone nord. Cela s'explique essentiellement, je crois, par le fait que

22. Cette couverture radar a été financée essentiellement par l'Université de Sydney et le Comité Mékong. C. Pottier et R. Fletcher, "The Gossamer City: seeing Greater Angkor », Museum International, $n^{\circ}$ 213-214, UNESCO, mai 2002, Paris, p. 23-27.

23. M. Donald Cooney, président de Concept Aviation.

24. Cette stèle a été découverte le 29 janvier 2002 par MM. Vân Sary et Koy au Prasat Phnom Dei (LL612, Phum Phluong, Khum Léang Dai, Srok Angkor Thom), dans l'aile sud de la salle centrale cruciforme du gopura oriental de première enceinte. Elle a été ramenée quelques jours plus tard au dépôt de la Conservation d'Angkor où elle est désormais inventoriée sous le $\mathrm{n}^{\circ} 1427$. 
FIG. 7. - Cartographie archéologique préliminaire avec la zone nord d'Angkor (EFEO/USYD/APSARA 2000-2003).

cette région plus éloignée n'a guère fait l'objet de recherches depuis 1910 alors qu'elle présente pourtant une concentration d'occupations anciennes au moins égale à celle du Sud. Dans ces conditions, il est assez délicat d'évaluer précisément ce que représentent les quelque 300 sites reconnus actuellement par rapport à l'ensemble de la zone concernée. Si l'on doit toutefois avancer une estimation, même très générale, elle pourrait représenter un peu plus de la moitié de la zone septentrionale d'Angkor que l'on pourra envisager d'étudier avec cette méthodologie.

\section{Mission archéologique française}

L'extension d'une approche horizontale de la recherche sur l'ensemble de la périphérie d'Angkor constitue la poursuite de l'étude réalisée de 1992 à 1999, avec une méthodologie renforcée et des moyens et des outils novateurs. Il m'a semblé toutefois 

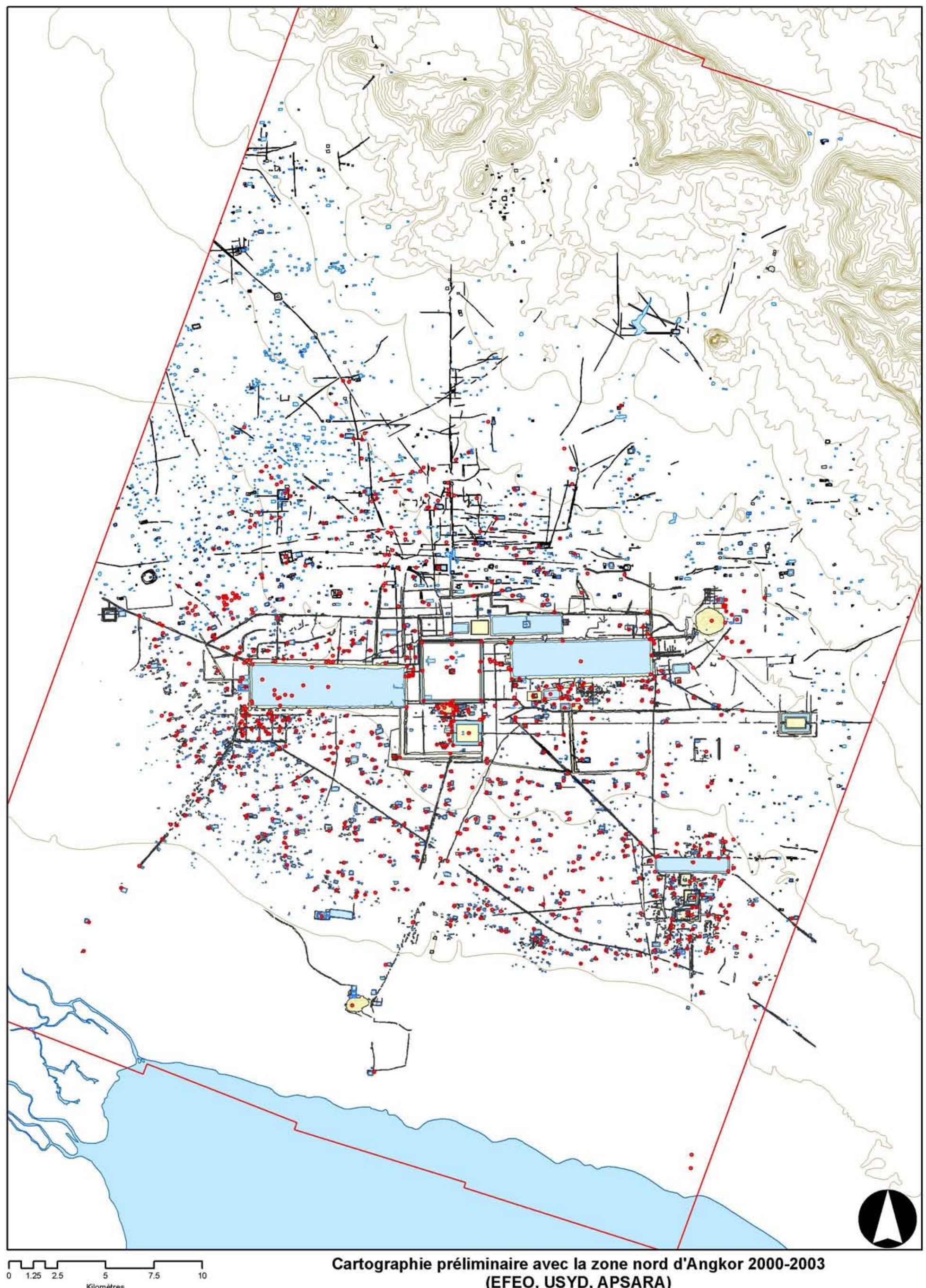

Cartographie préliminaire avec la zone nord d'Angkor 2000-2003 (EFEO, USYD, APSARA)

Les sites vérifiés au sol sont indiqués en rouge. 
nécessaire d'asseoir en parallèle cette approche grâce à des investigations en profondeur, ne serait-ce que pour confirmer la validité des hypothèses émises jusqu'alors. On ne pouvait donc pas faire l'impasse sur une approche verticale, en particulier par la confrontation de ces analyses spatiales avec les enseignements de la fouille stratigraphique.

Grâce à l'aide financière de la commission archéologique du ministère des Affaires étrangères, j'ai eu l'opportunité d'organiser en étroite collaboration avec l'autorité cambodgienne APSARA une Mission archéologique franco-khmère sur l'Aménagement du Territoire angkorien (MAFKATA). Avalisée par le Comité international de Coordination sur Angkor (CIC) en juin 2000 , cette mission archéologique a commencé dès la fin de cette même année ${ }^{25}$.

Outre l'aide à la formation d'archéologues cambodgiens, les objectifs de la mission portent sur l'étude verticale de plusieurs sites-clés pour les problématiques territoriales. Il pourrait certes sembler paradoxal d'envisager des fouilles alors que la problématique de recherche couvre une aire de $3000 \mathrm{~km}^{2}$. Mais cette disproportion entre une aire d'investigation gigantesque et les zones fouillées forcément réduites n'est qu'apparente. D'une part, la mission archéologique vise, à travers la réalisation de fouilles et de sondages ponctuels sur un nombre limité de sites de la région sud d'Angkor, à recueillir des éléments permettant d'évaluer, éventuellement de valider et de préciser certaines hypothèses territoriales formulées lors des recherches horizontales déjà réalisées sur cette zone. Les sites sont donc sélectionnés et choisis, d'après cette analyse spatiale globale, pour leur potentiel à caractériser certaines phases d'aménagements, certaines périodes d'occupations ou certains moments de ruptures dans l'histoire de l'évolution des aménagements territoriaux. Il s'agit ainsi d'une série d'interventions très ponctuelles vu l'ampleur du site d'Angkor, mais en des lieux particulièrement significatifs dont la pertinence est suggérée par le recoupement d'analyses à diverses échelles. D'autre part, les travaux réalisés avant 1970 montrent

25. Les quatre missions qui se sont déroulées ont vu la participation des archéologues suivants, Alexandrine Guérin [Lyon II-MOM-CNRS], Eric Llopis [INRAP-Cra Sophia Antipolis], Heng Than, Khieu Chan, Im Sokrithy [APSARA], Pierre Bâty et Annie Bolle [INRAP], Fabrice Demeter [Collège de France], Ernelle Berliet [Doctorante à Paris III] et les étudiantes Seila Yam et Tessa Simone Boer-Mah. 
que la région sud d'Angkor présente une complexité chronologique unique, les premières installations humaines pouvant remonter au néolithique et les capitales du royaume khmer s'y étant succédé quasiment sans discontinuité entre le début du IX et le $\mathrm{XV}^{\mathrm{c}}$ siècle. Alors que d'autres recherches archéologiques étudient actuellement certaines phases de l'époque angkorienne, il m'a semblé que les périodes en amont restaient particulièrement méconnues bien qu'elles soient essentielles pour comprendre l'origine et l'élaboration des systèmes d'aménagements qui se développeront à partir de la fin du $\mathrm{IX}^{\mathrm{e}}$ siècle. La mission a donc été dévolue à cette première phase, dite communément pré-angkorienne, où l'on pense pouvoir suivre l'apparition des principes urbains et des aménagements représentatifs des premières phases d'occupations. Cette phase court depuis les installations des fondations pré-angkoriennes sur des sites protohistoriques jusqu'à l'apparition de la cité angkorienne et à son développement territorial systématique au IX $\mathrm{X}^{\mathrm{e}}$ siècle. Toutefois, le choix des sites fouillés permet aussi, indirectement, d'aborder des problématiques territoriales relatives aux périodes postérieures, comme celles des aménagements hydrauliques du $\mathrm{XI}^{\mathrm{e}}$ siècle ou de l'évolution de la forme urbaine jusqu'au XII siècle. En effet, certains sites représentatifs des périodes primitives apportent aussi des éléments de compréhension primordiaux pour démêler les phases ultérieures du palimpseste que constitue la région d'Angkor.

Sites fouillés. Les trois premières campagnes de fouilles ont concerné cinq sites (dont deux inédits) dans la région du baray occidental, présumés être représentatifs des phases d'occupations pré-angkoriennes et d'aménagements territoriaux postérieurs. Les rapports remis à la commission archéologique du ministère des Affaires étrangères présentent le détail des opérations réalisées et les éléments de conclusion qu'elles apportent pour la compréhension des occupations des sites sondés ainsi que pour celle de divers faits territoriaux. Je préciserai seulement ici quelques éléments majeurs mis au jour sur ses cinq sites pré-angkoriens : Vat Khnat, Kôk Ta Sien (inédit), Prei Khmeng, Kôk Phnœu (inédit) et Aak Yum. Les résultats préliminaires permettent d'apporter de nouveaux éléments de compréhension relatifs aux diverses problématiques et quelques éléments de confirmations aux hypothèses territoriales émises préalablement. Par exemple, 
la découverte de la première nécropole protohistorique à Prei Khmeng (fig. 8, 9 et 10) offre une spectaculaire illustration de la superposition des premières occupations humaines et un cas prometteur pour y suivre une éventuelle continuité des installations lors de la période de «l'hindouisation». A Prei Khmeng comme sur la plupart des autres sites sondés, nous avons pu aussi étudier des vestiges de la période pré-angkorienne, qu'il s'agisse de structures architecturales comme à Vat Khnat, Kôk Ta Sien (fig. 11) ct Aak Yum, mais aussi des niveaux d'occupations domestiques et d'habitats. Certains apportent déjà des éléments qui confirmeraient cette dissociation en deux périodes pré-angkoriennes que j'avais cru pouvoir dégager par l'analyse spatiale. De même, les sondages à Vat Khnat, Kôk Ta Sien et Kôk Phnœu supportent mes hypothèses relatives à la configuration ouverte des établissements urbains pré-angkoriens et à l'organisation d'un vaste réseau hydraulique associé au baray occidental.

En complémentarité des premières campagnes, celle de 2004 a engagé l'étude de quelques sites similaires à Rolûos qui constitue une autre zone de la région d'Angkor où l'on peut suivre aussi les premières phases d'aménagement et l'apparition des principes urbains qui caractérisent l'histoire de la capitale angkorienne jusqu'au $\mathrm{XII}^{\mathrm{e}}$ siècle. En un premier temps, nous avons limité notre intervention à ces deux sites-clés que constituent Bakong et Trapéang Phong, afin d'optimiser l'interprétation des informations que les fouilles nous apportent. Enfin, la sécheresse exceptionnelle de cette année nous a permis de mettre au jour sur un site inédit situé dans le baray occidental, Koh Ta Méas, de remarquables vestiges d'installations préhistoriques dont une nouvelle nécropole associée.

Les fouilles, en particulier la découverte de la nécropole et de riches niveaux d'habitat à Prei Khmeng, ont aussi donné l'occasion d'engager des études spécifiques - en premier lieu l'élaboration d'une typo-chronologie des céramiques menée par $\mathrm{M}^{\mathrm{lle}} \mathrm{A}$. Guérin - et de développer des collaborations avec divers instituts internationaux. Ainsi plusieurs séries de datations au $\mathrm{C}^{14}$ ont été confiées au Dr Mike Barbetti, directeur du NWG Macintosh Centre for Quaternary Dating de l'Université de Sydney ${ }^{26}$.

26. U. Zoppi, M. Barbetti, R. K. Chhem, R. Fletcher, Q. Hua, M. Watanasak et C. Pottier, "The contribution of 14C AMS dating to the greater Angkor archaeological project », Nuclear Instruments and Methods in Physics Research Section B : Beam Interactions with 


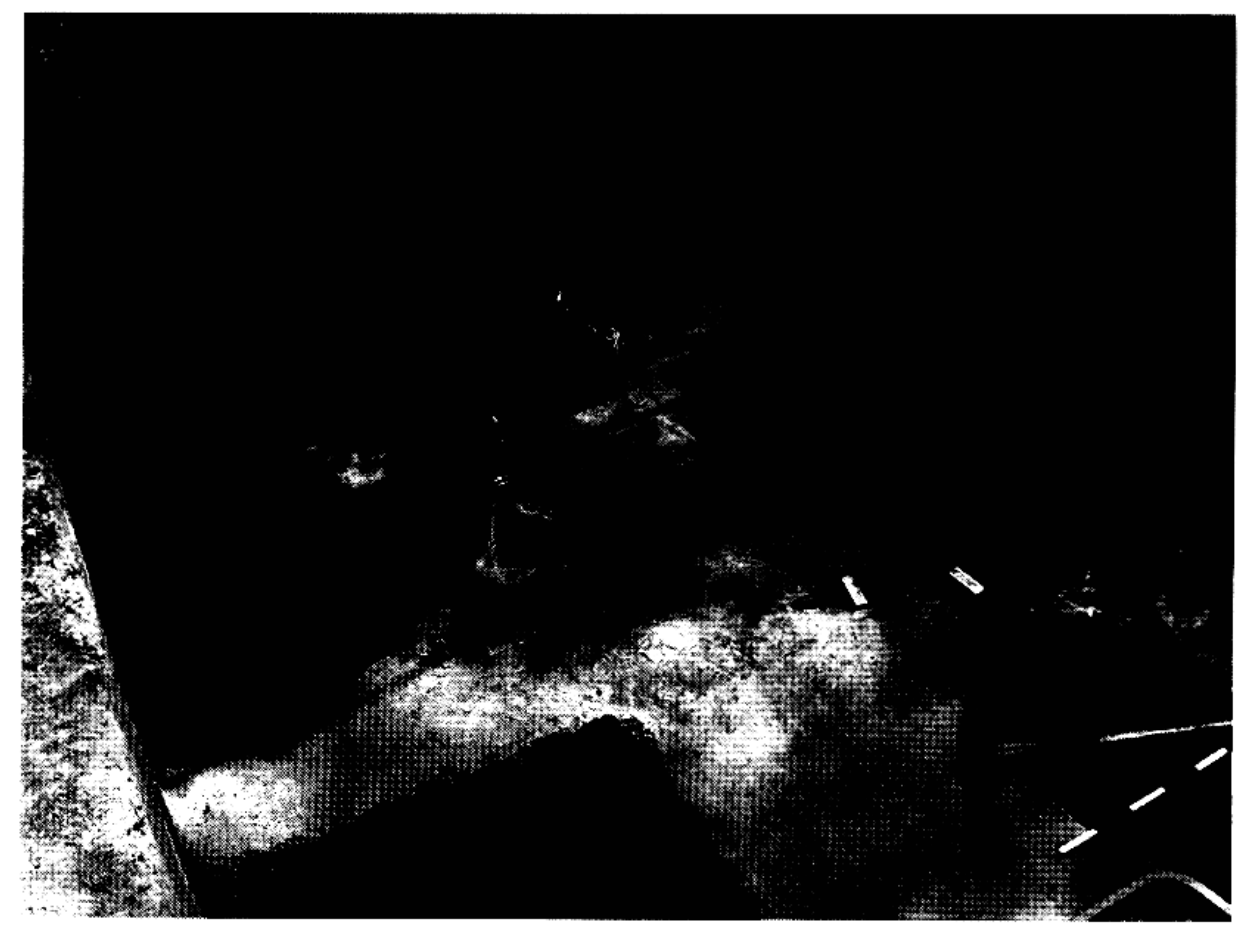

FIG. 8. - Sondage en cours à Prei Khmeng (2003). Noter la tombe à gauche et les niveaux d'habitats.

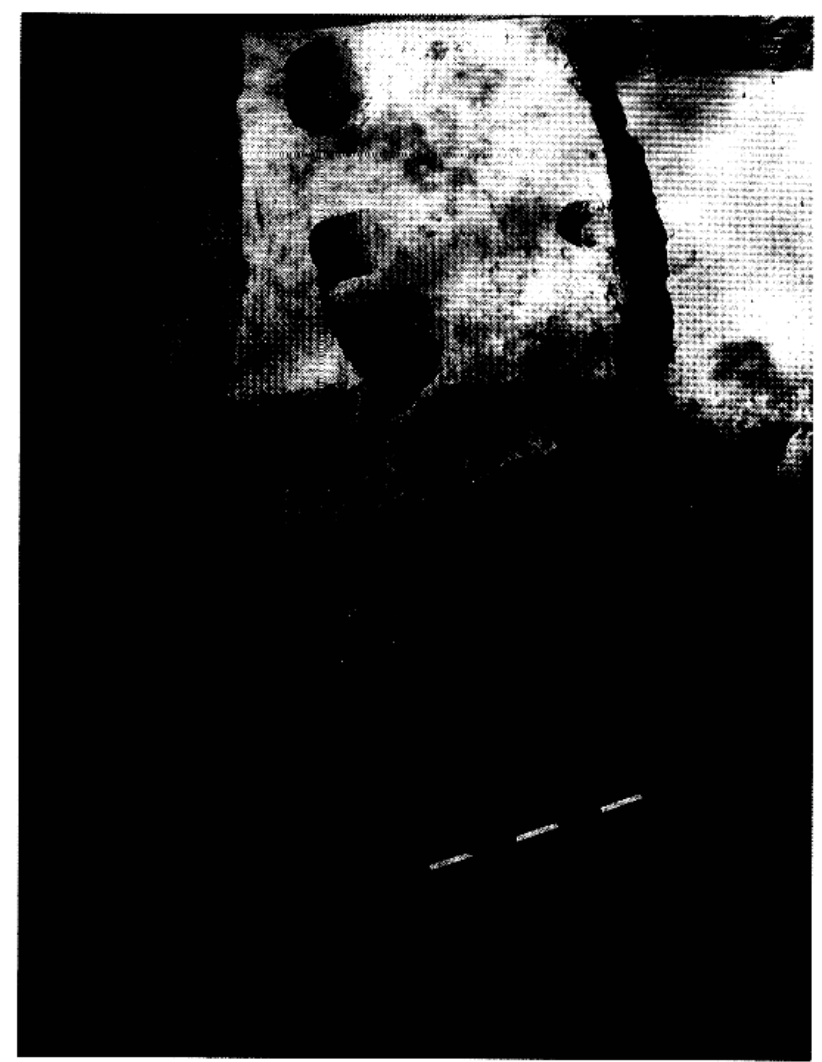

FIG. 9. - Sépulture à Prei Khmeng (2003). 





D'autre part, les premiers ossements humains ont été confiés au Dr. Rethy Kieth Chhem, alors directeur de la section Imagerie squelettique à l'Hôpital national universitaire de Singapour, qui a spécifiquement organisé un «Osteoarchaeology Research Group » pour procéder gracieusement à l'ensemble des examens nécessaires des deux squelettes envoyés (études anatomo-paléopathologique et radiologique, extraction d'ADN... $)^{27}$. Enfin, le $\mathrm{D}^{\mathrm{r}}$ Manas Watanasak, professeur à Mahidol University, Thaïlande, a réalisé une première recherche palynologique sur un échantillon associé à une tombe. Ces travaux se poursuivent actuellement avec le $\mathrm{D}^{\mathrm{r}}$ Dan Penny de l'Université de Sydney, engagé dans l'étude des changements paléo-environnementaux de la région ${ }^{28}$.

\section{Ouvertures}

Au terme de cette présentation, je souhaiterais souligner quelques traits qui me semblent caractériser cette recherche et son évolution. Initiée à l'occasion d'une étude pour la protection patrimoniale dans la région d'Angkor, et en marge de la direction de chantiers de restauration, cette recherche sur l'aménagement du territoire angkorien s'est développée depuis 1993 sur plusieurs axes. On a indiqué plus haut comment la première phase s'était orientée vers des investigations de surface dont la zone d'étude s'est accrue depuis au fur et à mesure de l'amélioration des conditions d'accessibilité. Ce développement horizontal s'est aussi accompagné d'une complexité croissante correspondant à l'affinement de la méthodologie, et plus spécifiquement à celui de la télédétection qui a élargi son catalogue de formes en intégrant des sites distincts des archétypes les plus courants. Cette complexité est aussi à mettre en relation tant avec l'expérience de terrain acquise sur plusieurs années de prospections régulières, qu'avec la facilité d'accès aux documentations existantes, en par-

Materials and Atoms, vol. 223-224, Proceedings of the Ninth International Conference on Accelerator Mass Spectrometry, août 2004, p. 681-685.

27. Rethy K Chhem, Sudhakar K Venkatesh, Shih-chang Wang, Kit-Mui Wong, Frank J. Ruhli, Edwin PY Siew, Kyle Latinis et C. Pottier, « Multislice computed tomography of the two 2000-year-old skeletons in a soil matrix from Angkor, Cambodia », Canadian Association of Radiologists Journal, vol. 55, No. 4, octobre 2004, Ottawa, p. 235-241.

28. D. Penny et al. «A palynological record... » (à paraître). 


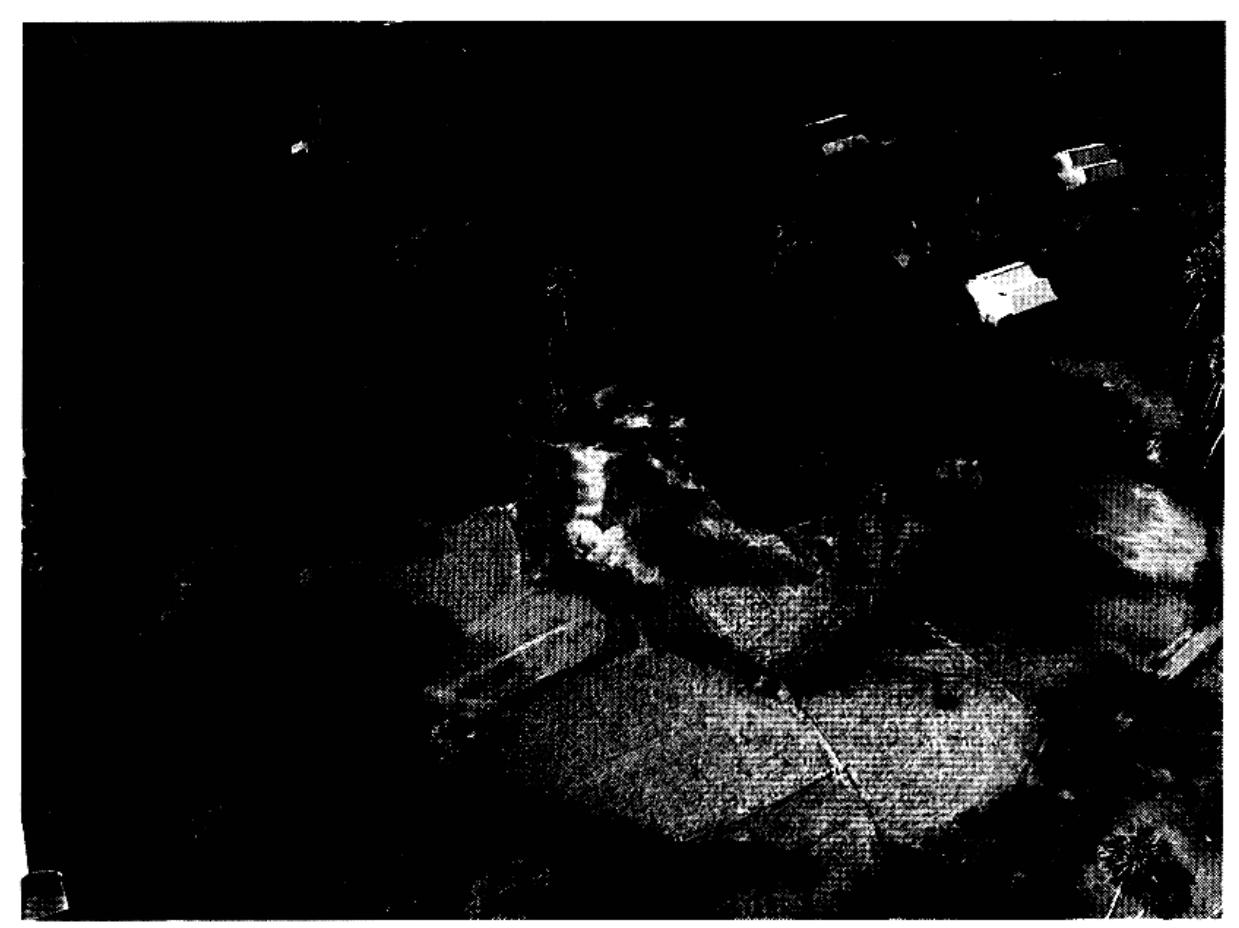

Fig. 10. - Le tertre de Prei Khmeng vu du nord-est. Noter sa douve périphérique (en rizière), sa chaussée orientale et les terre-pleins latéraux où est installé l'habitat actuel.

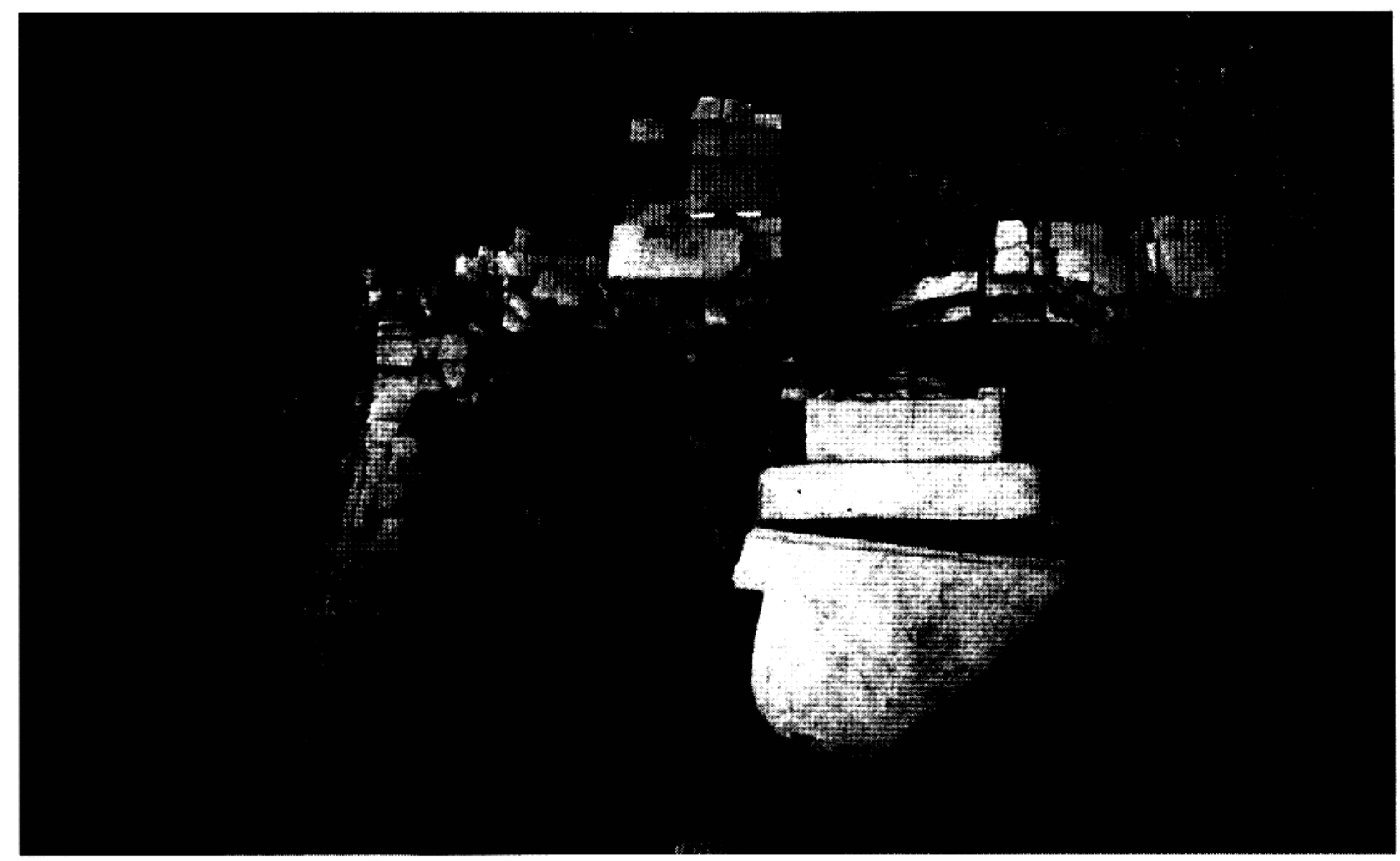

FIG. 11. - Emmarchement axial oriental du sanctuaire préangkorien découvert à Kôk Ta Sien (2001). 


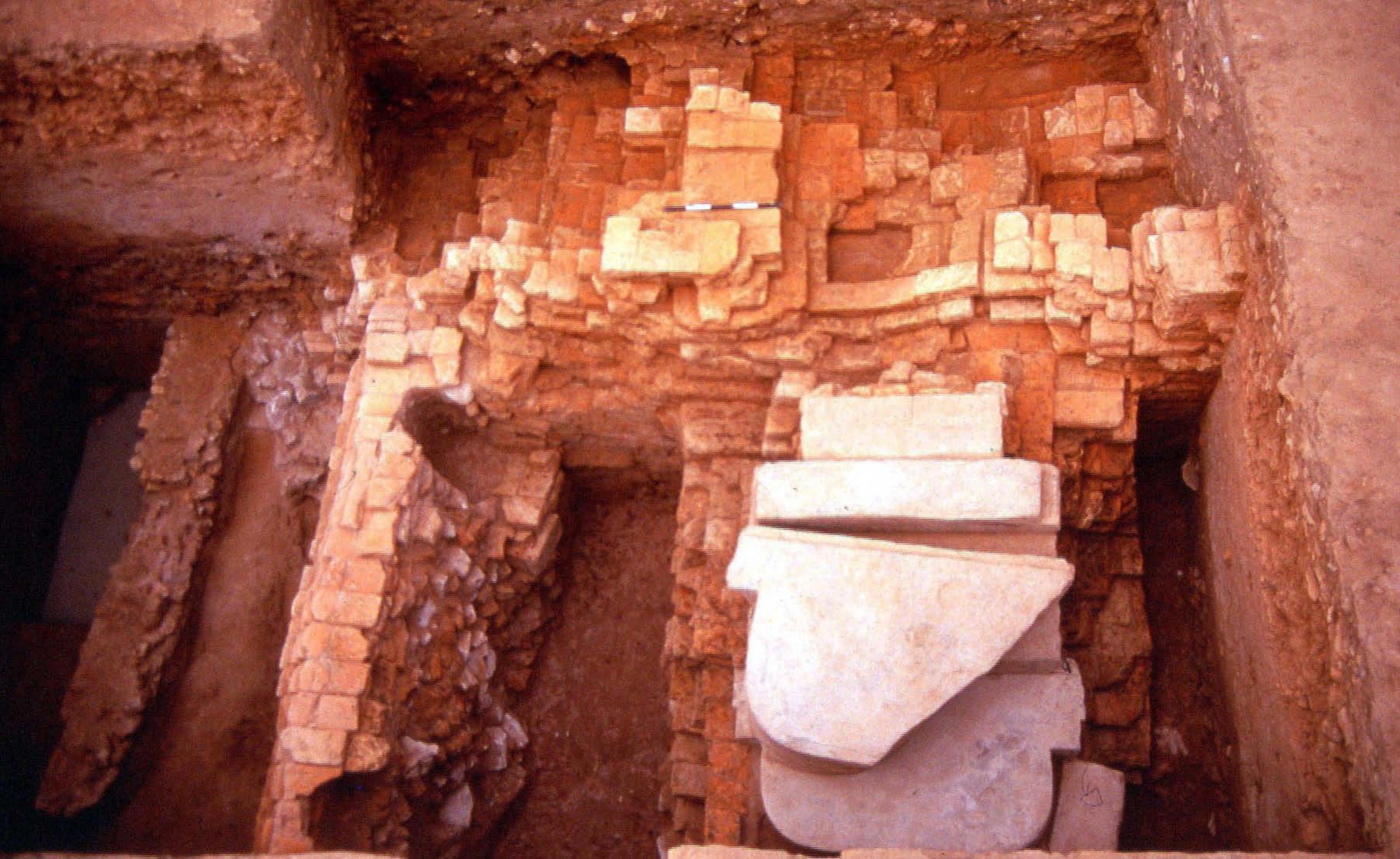


ticulier grâce au travail réalisé depuis 1990 par l'ÉFEO sur ses archives parisiennes relatives à Angkor. Ces travaux d'indexation, de saisie, de catalogage et de microfilmage ont permis un accès aisé aux informations, même depuis Siem Reap, et la réalisation d'une base de données des sites archéologiques synthétisant un maximum d'informations. En parallèle à cet accroissement de données, la recherche a aussi pu bénéficier - ou parfois se doter - de nouvelles sources d'informations : une nouvelle couverture photographique, complémentaire et de meilleure qualité que les anciennes des années 1950 , puis des images satellitaires, et encore plus récemment une large couverture radar de bonne résolution en trois dimensions.

La recherche spatiale s'est aussi attachée à développer de nouveaux outils d'analyse. Certains sont purement techniques (télédétection stéréoscopique systématique, intégration des cartes et des bases de données dans un SIG) alors que d'autres concernent plus directement l'élargissement de la notion même d'aménagement ancien aux éléments topographiques et parcellaires, ouvrant la voie à des études de chronologies relatives basées sur des relations morphologiques permettant une vision à la fois plus fine et plus globale des installations anciennes.

L'accroissement des informations obtenues par cette approche horizontale est donc particulièrement sensible ct, unc fois couplées avec les autres sources écrites, monumentales ou iconographiques, elles permettent l'élaboration d'hypothèses éclairant sensiblement la compréhension de l'histoire des installations dans la région d'Angkor, leur organisation et leur fonctionnement. Mais si l'accès et l'intégration des informations d'ordre spatial fournissent un cadre d'analyse complémentaire aux autres sources disponibles, ils ne permettent aucunement pour l'instant de s'affranchir de travaux archéologiques au sol.

L'approche verticale apparaît donc encore indissociable. Elle fournit en premier lieu une vérification de la méthode spatiale élaborée. C'est bien sûr le cas des prospections de terrains qui apportent une importante source d'informations souvent nouvelles, d'autant que de très nombreux pillages et fouilles illicites s'étaient multipliés dans la région d'Angkor au début des années 1990 , mettant au jour de nombreux artefacts inćdits. Ces prospections permettent aussi de recueillir des éléments nouveaux qui dépassent souvent la simple confirmation de l'identification de vestiges archéologiques, leur observation directe contribuant 
parfois à confirmer dans une certaine mesure les problématiques historiques globales par la mise en évidence d'éléments datés. Cette première phase de terrain concourre donc notablement à l'établissement d'une première vue d'ensemble, constituant un préalable à une investigation archéologique plus en profondeur. Celle-ci s'est développée depuis quatre ans en sélectionnant en premier lieu les problématiques relatives aux premières installations humaines de la pćriode historique dans la région. C'est sur cette période et en ces lieux que l'on espère pouvoir suivre l'apparition de modèles d'aménagements qui seront développés à la période angkorienne et qui caractériseront le fait urbain angkorien. Les prospections et l'analyse spatiale réalisées en amont fournissent alors le contexte et les problématiques à partir desquelles peuvent être implantées les opérations de fouilles, dans des sites potentiellement révélateurs non seulement de configurations ou d'époques spécifiques, mais aussi d'évolutions remarquables que l'on observe en d'autres lieux de la région d'Angkor.

Les campagnes de fouilles réalisées ne constituent donc pas uniquement une «simple » vérification. Elles offrent aussi, outre la richesse des informations recueillies, l'occasion de constituer des bases stratigraphiquement référencées sur lesquelles pourront à l'avenir s'appuyer par exemple l'étude du matériel collecté en surface lors des prospections cffcctuées dans la région, tant dans une perspective diachronique que dans le cadre d'une étude de la civilisation matérielle à l'échelle d'Angkor. Les investigations verticales ne constituent donc pas un terminus, mais au contraire une ouverture et un approfondissement en vue de poursuivre et développer l'analyse spatiale horizontale de l'aménagement du territoire angkorien.

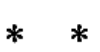

MM. Jean-François JARRIGE, Jean-Pierre MAHÉ, M $^{\text {me }}$ Colette CAILlat, MM. Azedine BESCHAOUCH, associé étranger de l'Académie, et Gilbert DAGRON interviennent après cette communication. 